\title{
Sulle funzioni epicicloidali e loro applicazione ad alcuni problemi di Fisica matematica.
}

\author{
Memoria di Cataldo Agostinelli (a Torino).
}

Sunto. - Si considera l'equazione differenziale alle derivate parziali del $2^{\circ}$ ordine in due variabili $\rho, \varphi$ (coordinate polari), che è la trasformata dell' equazione delle vibrazioni di una membrana elastica mediante le formule che dànno la rappresentazione conforme del campo piano racchiuso da una epicicloide con $\mathrm{n}-1$ lobi $(\mathrm{n}>1)$, con soli flessi, sopra un cerchio di raggio unitario. Si costruiscono quelle soluzioni di questa equazione, che vengono chiamate funzioni epicicloidali, le quali si riducono al prodotto di una funzione di Basse di prima specie di un dato ordine intero $\mathrm{k}$ per $\cos \mathrm{k} \varphi$, o per $\operatorname{sen} \mathrm{k} \varphi$, quando l'epicicloide tende a un cerchio.

Per mezzo di esse si risolvono alcuni problemi ai limiti che intervengono in quello delle vibrazioni di una membrana elastica con contorno epicicloidale fisso, in quello della propagazione guidata di onde elettromagnetiche in un tubo cilindrico indefinito a sezione epicicloidale riempito di dielettrico omogeneo isotropo, e in quello della conduzione del calore in un cilindro epicicloidale omogeneo indefinito che irradia calore in un ambiente esterno a temperatura castante.

\section{\& 1. Introduzione.}

1. I problemi relativi alle vibrazioni di una membrana con contorno epicicloidale fissata al suo orlo, alla conduzione del calore in un cilindro omogeneo di sezione epicicloidale, alla propagazione guidata di onde elettromagnetiche in un tubo cilindrico di sezione anch' esso epicicloidale, ecc., conducono alla considerazione di solazioni di una determinata equazione differenziale del $2^{\circ}$ ordine alle derivate parziali, con assegnate condizioni al contorno, soluzioni che danno luogo a nuove trascendenti che già in una nota lincea (') chiamai funzioni epicioloidali,

Della determinazione esplicita di dette funzioni, la cui importanza è legata ai problemi di Fisica matematica a cui ho accennato, mi occupo ora in questo lavoro,

Il campo piano in cui esse sono definite è limitato da una epicícloide $E$ ottenuta facendo rotolare un cerchio $\gamma$ di centro $C$ e di raggio $a$, esterna.

(1) C. AgostinelLi, Sulla determinazione degli autovalori nel problema delle vibrazioni di una membrana con contorno epicicloidale fisso, "Rendiconti dell'Accademia Nazionale dei Lincei ", serie VIII, vol. VII, fasc. 6, (1949). Vedi anche: A. Pignedoli, Frequenze di vibrazione di una membrana elastica a contorno epicicloidale fisso, "Annali di Matematica". (4), 30, (1949), 231-307. Idem, Su una equazione differenziale che si presenta nel problemá pelle vibrazioni di una membrana a contorno epicicloidale fisso, Atti del Sem. Matem. e fis. dell'Univ. di Modena », vol. IV, (1949.50). 
mente sopra un altro cerchio $\Gamma$ di raggio $(n-1) a,(n$ intero $>1)$, col centro $O$ nell' origine di un sistema di assi cartesiani ortogonali $O(x y)$. Supposto che la posizione iniziale $C_{0}$ di $C$ sia sutl' asse $O x$ e che un punto $M$ interno a $\gamma$ e rigidamente collegato con $\gamma$, sia pure inizialmente in una posizione $M_{0}$ di coordinate $(n a+b, 0)$, con $0<b<a$, dopo la rotazione del punto $C$ intorno ad $O$ di un angolo $\varphi$, le coordinate di $M$, che descrive una epicicloide chiusa $E$ con $n-1$ lobi e con soli flessi, saranno

$$
x=n a \cos \varphi+b \cos n \varphi, \quad y=n a \operatorname{sen} \varphi+b \operatorname{sen} n \varphi .
$$

Inoltre le equazioni che dànno la rappresentazione conforme del campo interno alla epicicloide $E$ descritta dal punto $M$, sul campo interno ad una circonferenza di raggio $\rho=1$, risultano

$$
x=n a \rho \cos \varphi+b \rho^{n} \cos n \varphi, \quad y=n a \rho \operatorname{sen} \varphi+b \rho^{n} \operatorname{sen} n \varphi .
$$

2. Nel caso di una membrana con contorno epicicloidale definito dalle $\left(1_{1}\right)$. fissa al suo orlo, lo spostamento $w$ di un suo punto $P(x, y)$, normalmente al suo piano, per effetto della sua vibrazione, soddisfa alla nota equazione di D'ALEMBERT

$$
\frac{\partial^{2} w}{\partial x^{2}}+\frac{\partial^{2} w}{\partial y^{2}}-\frac{1}{V^{2}} \frac{\partial^{2} w}{\partial t^{2}}=0, \quad(V=\text { costante })
$$

la quale, quando si ponga

$$
w=u(x, y) \operatorname{sen}(\lambda V t+\alpha),
$$

con $\lambda, \alpha$ costanti, porge, per la determinazione della funzione $u(x, y)$ l'equazione

$$
\frac{\partial^{2} u}{\partial x^{2}}+\frac{\partial^{2} u}{\partial y^{2}}+\lambda^{2} u=0
$$

con la condizione che la $u$ si annulli al contorno.

Trasformando l' equazione $\left(3_{1}\right)$ mediante le $\left(2_{1}\right)$ essa diventa

(4) $\quad \frac{\partial^{2} u}{\partial \rho^{2}}+\frac{1}{\rho} \frac{\partial u}{\partial \rho}+\frac{1}{\rho^{2}} \frac{\partial^{2} u}{\partial \varphi^{2}}+\lambda^{2} n^{2} a^{2}\left[1+2 c \rho^{n-1} \cos (n-1) \varphi+c^{2} \rho^{2(n-1)}\right] u=0$,

ove è $c=\frac{b}{a}<1$. Il problema delle vibrazioni della membrana epicicloidale si riduce allora a quello di determinare delle soluzioni della $\left(4_{1}\right)$, regolari nel campo interno al cerchio di raggio $\rho=1$, e che si annullano per $\rho=1$.

3. Se consideriamo ora la propagazione di onde elettromagnetiche in un tubo cilindrico indefinito, la cui sezione retta abbia per profilo l'epicicloide $E$ dianzi definita, supposto riempito di dielettrico omogeneo e isotropo di costante dielettrica $\varepsilon$ e di permeabilità nagnetica $\mu$, i vettori $\vec{E}, \vec{H}$, che rappresentano rispettivamente il campo elettrico e il campo magnetico entro il tubo, soddi- 
sfano, come si sa, alle seguenti equázioni di MAXwELL

$$
\operatorname{rot} \vec{H}=\frac{\varepsilon}{c_{0}} \frac{\partial \vec{E}}{\partial t}, \quad \operatorname{rot} \vec{E}=-\frac{\mu}{c_{0}} \frac{\partial \vec{H}}{\partial t},
$$

ove $c_{0}$ è la velocità della luce.

Sulla parete del tabo, supposto perfettamente conduttore, sarà nulla la componente tangenziale del campo elettrico, cioè

$$
\vec{E} \wedge \vec{n}=0,
$$

essendo $\vec{n}$ il versore della normale alla superficie.

Con riferimento a coordinate $\rho, \varphi, z$, ove $O z$ è l'asse del cilindro epicicloidale, il quadrato $d s^{2}$ dell' elemento lineare di spazio, in virtù delle $\left(2_{1}\right)$ risulta

$$
d s^{2}=n^{2} a^{2}\left[1+2 c \rho^{n-1} \cos (n-1) \varphi+c^{2} \rho^{2(n-1)}\right]\left(d \rho^{2}+\rho^{2} d \varphi^{2}\right)+d z^{2} .
$$

In questo sistema di coordinate, detto $P(\rho, \varphi, z)$ un punto interno a] tubo e indicando con

$$
\begin{array}{ll}
E_{\rho}=\vec{E} \times \frac{\partial P}{\partial \rho}, & E_{\varphi}=\vec{E} \times \frac{\partial P}{\partial \varphi}, \quad E_{z}=\vec{E} \times \frac{\partial P}{\partial z}, \\
H_{\rho}=\vec{H} \times \frac{\partial P}{\partial \rho}, \quad H_{\varphi}=\vec{H} \times \frac{\partial P}{\partial \varphi}, \quad H_{z}=\vec{H} \times \frac{\partial P}{\partial z}
\end{array}
$$

le componenti covarianti del campo elettrico e del campo magnetico, le equazioni vettoriali $\left(5_{1}\right)$ porgono il seguente sistema di equazioni scalari :

$$
\left\{\begin{array}{l}
\frac{1}{\rho}\left(\frac{\partial H_{z}}{\partial \varphi}-\frac{\partial H_{\varphi}}{\partial z}\right)=\frac{\varepsilon}{c_{0}} \frac{\partial E_{\rho}}{\partial t} \\
\rho\left(\frac{\partial H_{\rho}}{\partial z}-\frac{\partial H_{z}}{\partial \rho}\right)=\frac{\varepsilon}{c_{0}} \frac{\partial E_{\varphi}}{\partial t} \\
\frac{1}{n^{2} a^{2} R^{2} \rho}\left(\frac{\partial H_{\varphi}}{\partial \rho}-\frac{\partial H_{\rho}}{\partial \varphi}\right)=\frac{\varepsilon}{c_{0}} \frac{\partial E_{z}}{\partial t},
\end{array}\right.
$$

$$
\left\{\begin{array}{l}
\frac{1}{\rho}\left(\frac{\partial E_{z}}{\partial \varphi}-\frac{\partial E_{\varphi}}{\partial z}\right)=-\frac{\mu}{c_{0}} \frac{\partial H_{\rho}}{\partial t} \\
\rho\left(\frac{\partial E_{\rho}}{\partial z}-\frac{\partial E_{z}}{\partial \rho}\right)=-\frac{\mu}{c_{0}} \frac{\partial H_{\varphi}}{\partial t} \\
\frac{1}{n^{2} a^{2} R^{2} \rho}\left(\frac{\partial E_{\varphi}}{\partial \rho}-\frac{\partial E_{\rho}}{\partial \varphi}\right)=-\frac{\mu}{c_{0}} \frac{\partial H_{z}}{\partial t}
\end{array}\right.
$$

nelle quali per brevità si è posto

$$
R^{2}=1+2 c \rho^{n-1} \cos (n-1) \varphi+c^{2} \rho^{2(n-1)}=\frac{1}{n^{2} a^{2}}\left(\frac{\partial P}{\partial \rho}\right)^{2}=\frac{1}{n^{2} a^{2} \rho^{2}}\left(\frac{\partial P}{\partial \varphi}\right)^{2}
$$

mentre la condizione $\left(5_{1}^{\prime}\right)$ alla superficie diventa

$$
\bar{E} \wedge \frac{\partial P}{\partial \rho}=0
$$

cioè

$\left(8_{1}\right)$

$$
E_{\varphi}=0, \quad E_{z}=0, \quad \text { per } \rho=1 .
$$


Se supponiamo, come ordinariamente si fa in questo genere di questioni, che il campo elettromagnetico sia armonico rispetto al tempo, e indichiamo con $k c_{0}$ la pulsazione, e che si propaghi nel verso positivo dell'asse $z$ con una velocità costante $V=\frac{k c_{0}}{\alpha}$, possiamo porre, sotto forma complessa

$$
\vec{H}=\overrightarrow{\mathscr{H}}(\rho, \varphi) \cdot i\left(k c_{0} t-\alpha z\right), \quad \vec{E}=\overrightarrow{\mathcal{E}}(\rho, \varphi) e^{i\left(k c_{0} t-\alpha z z\right)}
$$

ove $\dot{H}(\rho, \varphi), \overrightarrow{\mathcal{G}}(\rho, \varphi)$ sono $\mathrm{i}$ vettori del campo elettromagnetico all' istante iniziale $(t=0)$, e per $z=0$.

Allora, se indichiamo ancora con $\mathfrak{H}_{\rho}, \mathfrak{H}_{\varphi}, \mathcal{H}_{z} ; \mathcal{E}_{\rho}, \mathcal{E}_{\varphi}, \mathcal{E}_{z}$ le componenti covarianti dei vettori $\overrightarrow{\mathfrak{K}}, \overrightarrow{\mathcal{E}}$, i sistemi di equazioni $\left(6_{1}\right),\left(6_{1}{ }^{\prime}\right)$ porgono;

$$
\begin{aligned}
& \left\{\begin{array}{l}
\frac{1}{\rho}\left(\frac{\partial \mathcal{H}_{z}}{\partial \varphi}+i \alpha \mathcal{H}_{\varphi}\right)=i k \varepsilon \mathcal{E}_{\rho} \\
-\rho\left(i \alpha \mathcal{H}_{\rho}+\frac{\partial \mathcal{H}_{z}}{\partial \rho}\right)=i k \varepsilon \mathcal{G}_{\varphi} \\
\frac{1}{n^{2} \alpha^{*} R^{2} \varphi}\left(\frac{\partial \mathcal{H}_{\varphi}}{\partial \rho}-\frac{\partial \mathcal{H}_{\rho}}{\partial \varphi}\right)=i k \varepsilon \mathcal{G}_{z}
\end{array}\right. \\
& \left(9_{i}\right)^{\prime}\left\{\begin{array}{l}
\frac{1}{\rho}\left(\frac{\partial \mathcal{G}_{z}}{\partial \varphi}+i \alpha \mathcal{G}_{\varphi}\right)=-i \mu k \mathcal{H}_{\rho} \\
\rho\left(i \alpha \mathcal{G}_{p}+\frac{\partial \mathcal{G}_{z}}{\partial \rho}\right)=i \mu k \mathcal{H}_{\varphi} \\
\frac{1}{n^{2} a^{z} R^{z} \rho}\left(\frac{\partial \mathcal{G}_{\varphi}}{\partial \rho}-\frac{\partial \mathcal{G}_{\rho}}{\partial \varphi}\right)=-i \mu k \mathcal{H}_{z},
\end{array}\right.
\end{aligned}
$$

e le condizioni al contorno $\left(8_{1}\right)$ diventano

$$
\mathfrak{G}_{\varphi}=0, \quad \mathfrak{G}_{z}=0, \quad \text { per } \rho=1 .
$$

Dalle prime due delle $\left(9_{1}\right)$ e delle $\left(9_{1}{ }^{\prime}\right)$ ponendo $\lambda^{2}=k^{2} \varepsilon \mu-\alpha^{2}$, si ricava

$$
\left(11_{t}\right)\left\{\begin{array}{l}
\mathscr{G}_{\rho}=-\frac{i}{\lambda^{2}}\left(k \mu \frac{\partial \mathcal{H}_{z}}{\partial \varphi}+\alpha \rho \frac{\partial \mathcal{E}_{z}}{\partial \rho}\right) \\
\mathscr{G}_{\varphi}=\frac{i}{\lambda^{2}}\left(k \mu \rho \frac{\partial \mathcal{H}_{z}}{\partial \rho}-\alpha \frac{\partial \mathcal{G}_{z}}{\partial \varphi}\right) \\
\mathscr{H}_{\rho}=\frac{i}{\lambda^{2} \rho}\left(k s \frac{\partial \mathscr{G}_{z}}{\partial \varphi}-\alpha \rho \frac{\partial \mathcal{H}_{z}}{\partial \rho}\right) \\
\mathscr{H}_{\varphi}=-\frac{i}{\lambda^{2}}\left(\alpha \frac{\partial \mathcal{H}_{z}}{\partial \varphi}+k \varepsilon \rho \frac{\partial \mathscr{G}_{z}}{\partial \rho}\right)
\end{array}\right.
$$

e sostituendo nelle rimanenti si ha che $\mathcal{G}_{z}$ ed $\mathscr{H}_{z}$ devono verificare la medesima equazione differenziale $\left(4_{1}\right)$, con condizioni al contorno che per le $\left(10_{1}\right)$ e la $2^{\text {a delle }}(11$,$) risultano$

$$
\mathfrak{G}_{z}=0, \quad k \mu \rho \frac{\partial \mathcal{H}_{z}}{\partial \rho}-\alpha \frac{\partial \mathfrak{G}_{z}}{\partial \varphi}=0, \quad \text { per } \rho=1 .
$$

Queste condizioni si possono rendere compatibili in due modi diversi: $1^{\circ}$ Ponendo identicamente $\mathscr{G}_{z}=0$, e quindi $E_{z}=0$ (onde di tipo magnetico). 
In questo caso la componente $\mathscr{H}_{z}$ dal campo elettromagnetico dovrà verificare l'equazione differenziale $\left(4_{1}\right)$ con la coudizione al contorno

$$
\frac{\partial H_{z}}{\partial \rho}=0, \quad \text { per } \rho=1 .
$$

Ottenuta la $\mathfrak{H}_{z}$ le rimanenti componenti del campo, per la $\left(11_{1}\right)$, saranno date dalle

$$
\left\{\begin{array}{l}
\mathscr{E}_{\rho}=-i \frac{k \mu}{\lambda^{2}} \frac{1}{\rho} \frac{\partial \mathcal{H}_{z}}{\partial \varphi}, \quad \mathscr{E}_{\varphi}=i \frac{k \mu}{\lambda^{2}} \rho \frac{\partial \mathcal{H}_{z}}{\partial \rho} \\
\mathscr{H}_{\rho}=-i \frac{\alpha}{\lambda^{2}} \frac{\partial \mathcal{H}_{z}}{\partial \rho}, \quad \mathscr{H}_{\varphi}=-i \frac{\alpha}{\lambda^{2}} \frac{\partial \mathcal{H}_{z}}{\partial \varphi} .
\end{array}\right.
$$

$2^{\circ}$ Ponendo identicamente $\mathfrak{H}_{z}=0$, ciò̀ $H_{z}=0$ (onde di tipo elettrico).

In tal caso la componente $\mathfrak{G}_{z}$ del campo elettromagnetico dovrà verificare l'equazione differenziale $\left(4_{1}\right)$ con la condizione ai limiti

$$
\mathfrak{E}_{z}=0, \quad \text { per } \rho=1 \text {. }
$$

Determinata la $\mathscr{G}_{z}$ le altre componenti del campo, in virtù della $\left(11_{1}\right)$ saranno date dalle

$$
\begin{aligned}
& \mathscr{E}_{p}=-i \frac{\alpha}{\lambda^{2}} \frac{\partial \mathcal{E}_{z}}{\partial \rho}, \quad \mathscr{E}_{\varphi}=-i \frac{\alpha}{\lambda^{2}} \frac{\partial \mathscr{E}_{z}}{\partial \varphi}, \\
& \mathcal{H}_{\rho}=i \frac{k \varepsilon}{\lambda^{2}} \frac{1}{\rho} \frac{\partial \mathfrak{G}_{\tilde{z}}}{\partial \varphi}, \quad \mathscr{H}_{\varphi}=-i \frac{k \varepsilon}{\lambda^{2}} \rho \frac{\partial \mathfrak{E}_{z}}{\partial \rho} .
\end{aligned}
$$

4. Consideriamo inïine il problema della conduzione del calore in un cilindro omogeneo indefinito a sezione epicicloidale, nell' ipotesi che la temperatura sia indipendente dalla coordinata $z$, e supponendo che irradi calore nell' ambiente esterno a temperatura costante uguale a zero.

La temperatura $U$ in ogni punto $P(x, y)$ interno al cilindro $\mathrm{e}$ in un istante generico $t$ dovrà soddisfare all' equazione differenziale

$$
\frac{\partial^{2} U}{\partial x^{2}}+\frac{\partial^{2} U}{\partial y^{2}}-\frac{1}{k_{0}} \frac{\partial U}{\partial t}=0
$$

ove $k_{0}$ è una costante positiva dipendente dalla densita del corpo e dalla conducibilità interna, mentre alla superficie deve aversi

$$
\frac{d U}{d n}+h U=0
$$

essendo $\frac{d U}{d n}$ la derivata normale (esterna) di $U$, ed $h$ un'altra costante dipen. dente dal potere emissivo alla superficie. 
Ponendo $U=u(x, y) e^{-\lambda 2 h_{0} t}$ la $\left(15_{1}\right)$ si riduce alla stessa equazione $\left(3_{1}\right)$ e la condizione $\left(16_{1}\right)$ diventa

$$
\frac{d u}{d n}+h u \equiv 0
$$

Mediante la trasformazione definita dalle equazioni $(2$,$) si ha che la$ funzione $u$ deve essere ancora soluzione dell' equazione differenziale $\left(4_{4}\right)$. In quanto alla condizione al contorno, poichè netto $\vec{n}$ il versore della normale esterna alla superficie, risulta

$$
\frac{d u}{d n}=\operatorname{grad} u \times \vec{n}=\operatorname{grad} u \times \frac{\partial P}{\partial \rho} / \bmod \frac{\partial P}{\partial \rho}=\frac{\partial u}{\partial \rho} /(\operatorname{na} R),
$$

la detta condizione diventa

$$
\frac{1}{n a R} \frac{\partial u}{\partial \rho}+h u=0, \quad \text { per } \rho=1
$$

ove il valore di $R$ è definito dalla $\left(7_{1}\right)$.

\section{§ 2. Le funzioni $\mathcal{G}^{(k)}$.}

1. Per la ricerca di soluzioni dell'equazione differenziale $\left(4_{i}\right)$, che permettano di risolvere i problemi precedenti, poniamo per semplicità

$$
r=\lambda n a \rho, \quad \gamma=\frac{c}{(\lambda n a)^{n-1}}
$$

e allora la detta equazione diventa

$$
\frac{\partial^{*} u}{\partial r^{2}}+\frac{1}{r} \frac{\partial u}{\partial r}+\frac{1}{r^{2}} \frac{\partial^{2} u}{\partial \varphi^{2}}+\left[1+2 \gamma r^{n-1} \cos (n-1) \varphi+\gamma^{2} r^{2(n-1)}\right] u=0
$$

che per $\gamma=0$, cioè $c=0$, si riduce alla

$$
\frac{\partial^{2} u}{\partial r^{2}}+\frac{1}{r} \frac{\partial u}{\partial r}+\frac{1}{r^{2}} \frac{\partial^{2} u}{\partial \varphi^{2}}+u=0
$$

che ammette soluzioni della forma, regolari per $r=0, J_{h}(r) \cos k \varphi, J_{k}(r)$ sen $k \varphi$, essendo $J_{k}(r)$ la funzione di Bessel di $1^{a}$ specie e di ordine $\mathrm{k}$, con $k$ intero positivo o nullo.

Proponiamoci allora di determinare soluzioni della $\left(2_{2}\right)$ che per $\gamma=0$ si riducano a $J_{k}(r) \cos k \varphi$, oppure $J_{k}(r)$ sen $k \varphi$, che indicheremo rispettivamente con $\mathcal{G}^{(k)}(r, \varphi, \gamma)$, ed $\mathfrak{G}^{*(k)}(r, \varphi, \gamma)$.

A queste funzioni, come pure ad ogni loro combinazione lineare a coefficienti costanti, daremo ora più propriamente il nome di funzioni epicicloidali. 
Incominciando dalle prime, se immaginiamo la $\mathfrak{o}^{(k)}$ sviluppata in serie di potenze di $\gamma$, avremo uno sviluppo della forma

$$
\mathcal{G}^{(k)}=J_{k}(r) \cos k \varphi+\sum_{1}^{\infty} \gamma^{j} \mathcal{G}_{j}{ }^{(k)}(r, \varphi) \text {. }
$$

Sostituendo nella $\left(2_{z}\right)$, e tenendo conto che la $J_{k}(r)$ soddisfa all'equazione differenziale

$$
\frac{d^{2} J_{k}}{d r^{2}}+\frac{1}{r} \frac{d J_{k}}{d r}+\left(1-\frac{k^{2}}{r^{2}}\right) J_{k}=0
$$

si ha

$$
\begin{aligned}
& {\stackrel{\Sigma}{\Sigma}, \gamma^{\prime}}^{\infty}\left(\frac{\partial^{2} \mathcal{G}_{j}^{(k)}}{\partial r^{2}}+\frac{1}{r} \frac{\partial \mathcal{G}_{j}{ }^{(k)}}{\partial r}+\frac{1}{r^{2}} \frac{\partial^{2} \mathcal{G}_{j}{ }^{(k)}}{\partial \varphi^{2}}+\mathcal{g}_{j}{ }^{(k)}\right)+2 \gamma \gamma^{n-1} J_{k}(r) \cos k \varphi \cdot \cos (n-1) \varphi+ \\
& +\gamma^{2} r^{2(n-1)} J_{k}(r) \cos k \varphi+2 r^{n-1} \cos (n-1) \varphi \cdot \sum_{2}^{\infty} \gamma^{j} \mathcal{G}_{j-1}^{(k)}+r^{2(n-1)} \sum_{3}^{\infty} \gamma^{j} \mathcal{G}_{j-2}^{(k)}=0 .
\end{aligned}
$$

Questa dovrà essere identicamente verificata qualunque sia $\gamma$; perciò uguagliando a zero i coefficienti delle successive potenze di $\gamma$, si ha il sistema

(I)

$$
\begin{aligned}
& \frac{\partial^{2} \mathcal{G}_{1}{ }^{(k)}}{\partial r^{2}}+\frac{1}{r} \frac{\partial \mathcal{G}_{1}{ }^{(k)}}{\partial r}+\frac{1}{r^{2}} \frac{\partial^{2} \mathcal{G}_{i}{ }^{(k)}}{\partial \varphi^{2}}+\mathcal{E}_{1}{ }^{(k)}+2 r^{n-1} J_{k}(r) \cdot \cos k \varphi \cdot \cos (n-1) \varphi=0, \\
& \frac{\partial^{2} \mathcal{G}_{2}{ }^{(k)}}{\partial r^{2}}+\frac{1}{r} \frac{\partial \mathcal{G}_{2}{ }^{(k)}}{\partial r}+\frac{1}{r^{2}} \frac{\partial^{2} \mathcal{G}_{2}{ }^{(k)}}{\partial \varphi^{2}}+\mathscr{G}_{2}{ }^{(k)}+r^{2(n-1)} J_{k}(r) \cos k \varphi+
\end{aligned}
$$

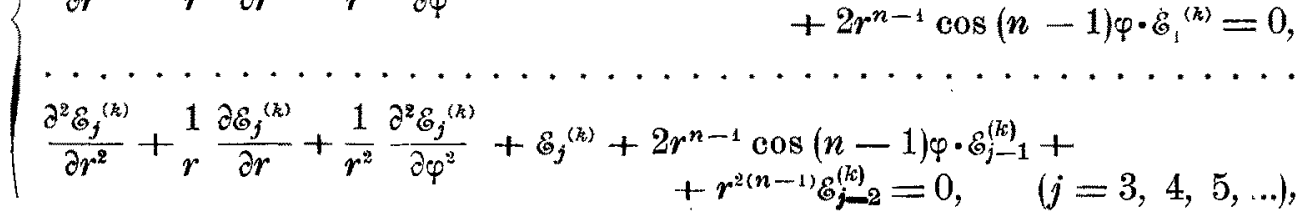

che consente di calcolare per via ricorrente le funzioni $\mathfrak{G}_{j}{ }^{(k)}$.

2. La prima delle (I) si può scrivere

$\frac{\partial^{2} \mathscr{E}_{1}{ }^{(k)}}{\partial r^{2}}+\frac{1}{r} \frac{\partial \mathcal{G}_{1}{ }^{(k)}}{\partial r}+\frac{1}{r^{2}} \frac{\partial^{2} \mathscr{G}_{1}{ }^{(k)}}{\partial \varphi^{2}}+\mathcal{E}_{1}{ }^{(k)}+r^{n-1} J_{k}(r)[\cos (k+n-1) \varphi+\cos (k-(n-1)) \varphi]=0$,

e ponendo

$$
\mathfrak{G}_{1}^{(k)}=\mathcal{E}_{11}^{(k)}(r) \cos (k+n-1) \varphi+g_{12}^{(k)}(r) \cos (k-i n-1) \| \varphi,
$$

si hanno per $\mathscr{E}_{11}^{(k)}(r)$ ed $\mathscr{E}_{12}^{(k)}(r)$ le equazioni differenziali

$$
\begin{aligned}
& \frac{d^{*} \mathcal{E}_{11}^{(k)}}{d r^{2}}+\frac{1}{r} \frac{d \mathcal{G}_{11}^{(k)}}{d r}+\left[1-\frac{(k+n-1)^{2}}{r^{2}}\right] \mathscr{E}_{11}^{(k)}+r^{n-1} J_{k}(r)=0, \\
& \frac{d^{2} \mathcal{E}_{12}^{(k)}}{d r^{2}}+\frac{1}{r} \frac{d \mathcal{E}_{12}^{(k)}}{d r}+\left[1-\frac{(k-n+1)^{2}}{r^{2}}\right] \mathscr{E}_{12}^{(k)}+r^{n-1} J_{k}(r)=0
\end{aligned}
$$


Cercando di queste soluzioni della forma

$$
\mathcal{E}_{11}^{(k)}=\Sigma_{0} A_{h}(r) J_{k-h}(r), \quad \mathcal{E}_{12}^{(k)}=\Sigma_{0} B_{h}(r) J_{k+h}(r),
$$

con $A_{h}(r), B_{h}(r)$ funzioni incognite di $r$, tenendo conto dell'equazione differenziale delle funzioni di Bessel e delle note relazioni ricorrenti che legano queste funzioni, si trova facilmente

$$
\begin{aligned}
& \mathfrak{E}_{11}^{(k)}=\frac{r^{n-1}}{4 n(k-1)}\left[J_{k}(r)+J_{k-2}(r)\right]=\frac{r^{n}}{2 n} J_{k-1}(r) \\
& \mathfrak{G}_{12}^{(k)}=-\frac{r^{n+1}}{4 n(k+1)}\left[J_{k}(r)+J_{k+2}(r)=-\frac{r^{n}}{2 n} J_{k+1}(r),\right.
\end{aligned}
$$

e pertanto

$\left(4_{2}\right) \quad \mathscr{G}_{1}{ }^{(k)}=\frac{r^{n}}{2 n}\left[J_{k-1}(r) \cdot \cos (k+n-1) \varphi-J_{k+1}(r) \cdot \cos (k-(n-1) \varphi]\right.$.

3. Sostituendo ora nella seconda delle (I) in luogo di $\mathscr{f}_{1}^{(k)}$ il valore trovato, si ha, per la determinazione della funzione $\mathfrak{G}_{2}{ }^{(h)}$, l'equazione differenziale

$$
\begin{aligned}
& \qquad \frac{\partial^{2} \mathcal{G}_{2}{ }_{2}(k)}{\partial r^{2}}+\frac{1}{r} \frac{\partial \mathcal{G}_{2}{ }^{(k)}}{\partial r}+\frac{1}{r^{2}} \frac{\partial^{2} \mathcal{G}_{2}{ }^{(k)}}{\partial \varphi^{2}}+{\mathcal{G}_{i}}^{(k)}+r^{2(n-1)} J_{k}(r) \cdot \cos k \varphi+ \\
& +\frac{r^{2 n-1}}{2 n}\left\{J_{k-1}(r) \cdot[\cos (k+2(n-1)) \varphi+\cos k \varphi]-J_{k+1}(r)[\cos k \varphi+\cos (k-2(n-1)) \varphi]\right\}=0, \\
& \text { e ponendo }
\end{aligned}
$$

$$
\mathcal{E}_{2}^{(k)}=\mathfrak{G}_{21}^{(k)}(r) \cos k \varphi+\mathfrak{G}_{22}^{(k)}(r) \cos (k+2(n-1)) \varphi+\mathfrak{G}_{23}^{(k)}(r) \cos (k-2(n-1)) \varphi,
$$

si ottiene il sistema

$$
\begin{aligned}
& \frac{d^{2} \mathcal{E}_{21}^{(k)}}{d r^{2}}+\frac{1}{r} \frac{d \mathcal{E}_{21}^{(k)}}{d r}+\left(1-\frac{k^{2}}{r^{2}}\right) \mathcal{E}_{21}^{(k)}+r^{2(n-1)} J_{k}+\frac{r^{2 n-1}}{2 n}\left(J_{k-1}-J_{k+1}\right)=0, \\
& \frac{d^{2} \mathfrak{E}_{22}^{(k)}}{d r^{2}}+\frac{1}{r} \frac{d \mathfrak{E}_{23}^{(k)}}{d r}+\left[1-\frac{(k+2(n-1))^{2}}{r^{2}}\right] \mathfrak{E}_{22}^{(k)}+\frac{r^{2 n-1}}{2 n} J_{k-1}=0, \\
& \frac{d^{*} \mathcal{E}_{23}^{(k)}}{d r^{2}}+\frac{1}{r} \frac{d \mathfrak{E}_{23}^{(k)}}{d r}+\left[1-\frac{(k-2(n-1))^{2}}{r^{2}}\right] \mathscr{E}_{23}^{(k)}-\frac{r^{2 n-1}}{2 n} J_{k+1}=0 .
\end{aligned}
$$

In virtù della formula ricorrente $J_{k}(r)=\frac{r}{2 k}\left(J_{k-1}+J_{k+1}\right)$, la prima di queste equazioni diventa

$$
\frac{d^{2} \mathcal{G}_{21}^{(k)}}{d r^{2}}+\frac{1}{r} \frac{d \mathfrak{E}_{21}^{(k)}}{d r}+\left(1-\frac{k^{2}}{r^{2}}\right) \mathfrak{E}_{21}^{(k)}+\frac{1}{2 k n} r^{2 n-1}\left[(n+k) J_{k-1}+(n-k) J_{k+1}\right]=0
$$


che ammette la soluzione

$$
\mathfrak{G}_{21}^{(k)}=-\frac{r^{2 n+1}}{8 n^{2} k}\left(J_{k-1}+J_{k+1}\right)=-\frac{r^{2 n}}{4 n^{2}} J_{k}(r) .
$$

Osserviamo ora che l'equazione in $\mathscr{G}_{22}^{(k)}$ si ottiene da quella in $\mathfrak{G}_{11}^{(k)}$, ponendo $k-1$ al posto di $k, 2 n$ al posto di $n$, e $2 n \mathcal{E}_{22}^{(k)}$ al posto di $\mathfrak{E}_{11}^{(k)}$. Ricordando il valore di $\mathfrak{G}_{11}^{(k)}$ si deduce percio

$$
\mathfrak{G}_{22}^{(k)}=\frac{r^{2 n}}{2 \cdot 4 n^{2}} J_{k-2}(r)
$$

Così pure l'equazione in $\mathcal{E}_{23}^{(k)}$ si ottiene da quella in $\mathscr{E}_{12}^{(k)}$ cambiando $k$ in $k+1, n$ in $2 n$, ed $\mathfrak{G}_{12}^{(k)}$ in $-2 n \varepsilon_{23}^{(k)}$; quindi

$$
\mathfrak{E}_{23}^{(k)}=\frac{r^{2 n}}{2 \cdot 4 n^{2}} J_{k+2}(r)
$$

Ne segue

$\left(5_{2}\right) \mathscr{G}_{2}{ }^{(k)}=\frac{r^{2 n}}{2 \cdot 4 n^{2}}\left[J_{k-2}(r) \cos (k+2(n-1)) \varphi-2 J_{k}(r) \cos k \varphi+J_{k+2}(r) \cos (k-2(n \quad 1)) \varphi\right]$.

Con calcoli perfettamente analoghi si ricava

$$
\begin{aligned}
\mathcal{E}_{3}{ }^{(k)}= & \frac{r^{3 n}}{2^{3} \cdot 3 ! n^{3}}\left\{J_{k-3}(r) \cdot \cos (k+3(n-1)) \varphi-J_{k+3}(r) \cos (k-3(n-1)) \varphi-\right. \\
& \left.\quad-3\left[J_{k-1}(r) \cos (k+n-1) \varphi-J_{k+1}(r) \cos (k-(n-1)) \varphi\right]\right\}, \\
\mathfrak{E}_{4}{ }^{(k)}= & \frac{r^{4 n}}{2^{4} \cdot 4 ! n^{4}}\left\{J_{k-4} \cos (k+4(n-1)) \varphi+J_{k+4} \cos (k-4(n-1)) \varphi-\right. \\
& \left.\quad-4\left[J_{k-2} \cos (k+2(n-1)) \varphi+J_{k+2} \cos (k-2(n-1)) \varphi\right]+6 J_{k} \cos k \varphi\right\} .
\end{aligned}
$$

4. Prendiamo ora in considerazione l' equazione generica del sistema (I), I calcoli precedenti mostrano che sarà in generale

$$
\mathcal{E}_{j}^{(k)}=r^{j n} F_{j}^{(k)}(r, \varphi)
$$

ove $\left.F_{j}{ }^{(k)}, r, \varphi\right)$ è somma di prodotti della forma $J_{k ; h}(r) \cos (k-h(n-1)) \varphi$, $J_{k-h}(r) \cos (k+h(n-1)) \varphi$. Sostituendo si ottiene per la funzione $F_{j}^{(k)}$ l'equazione differenziale

$$
\begin{aligned}
\frac{\partial^{2} F_{j}^{(k)}}{\partial r^{2}}+ & \frac{2 j n+1}{r} \frac{\partial F_{j}^{(k)}}{\partial r}+\frac{1}{r^{2}} \frac{\partial^{2} F_{j}^{(k)}}{\partial \varphi^{2}}+\left(1+\frac{j n^{2}}{r^{2}}\right) F_{j}^{(k)}+ \\
& +\frac{2}{r} \cos (n-1) \varphi \cdot F_{j-1}^{(k)}+\frac{1}{r^{2}} F_{j-2}^{(k)}=0
\end{aligned}
$$


la quale, per $j=2 h$ (pari), e per $j=2 h+1$ (dispari), porge

$(6$,

$$
\begin{aligned}
\frac{\partial^{2} F_{2 h}^{(k)}}{\partial r^{2}}+\frac{4 h n+1}{r} \frac{\partial F_{2 h}^{(k)}}{\partial r}+\frac{1}{r^{2}} \frac{\partial^{2} F_{2 h}^{(k r)}}{\partial \varphi^{2}}+ & \left(1+\frac{4 h^{2} n^{2}}{r^{2}}\right) F_{2 h}^{(k)}+ \\
& +\frac{2}{r} \cos (n-1) \varphi \cdot F_{2 h-1}^{(k)}+\frac{1}{r^{2}} F_{2(h-1)}^{(k)}=0, \\
\frac{\partial^{2} F_{2 h+1}^{(k)}}{\partial r^{2}}+\frac{2(2 h+1) n+1}{r} \frac{\partial F_{2 h+1}^{(k)}}{\partial r}+ & \frac{1}{r^{2}} \frac{\partial^{2} F_{2 h+1}^{(k)}}{\partial \varphi^{4}}+\left(1+\frac{(2 h+1)^{2} n^{2}}{r^{2}}\right) F_{2 h+1}^{(k)}+ \\
& +\frac{2}{r} \cos (n-1) \varphi \cdot F_{2 h}^{(k)}+\frac{1}{r^{2}} F_{2 h-1}^{(k)}=0 .
\end{aligned}
$$

Ponendo

$$
\begin{aligned}
F_{2 h}^{(k)}=a_{h 0} J_{k}(r) \cos k \varphi+\sum_{i}^{h}\left[a_{h i} J_{k-2 i}(r) \cos (k+2 i(n-1)) \varphi+\right. & \\
& \left.+a_{h i}^{\prime} J_{k+2 i}(r) \cos (k-2 i(n-1)) \varphi\right], \\
F_{2 h+1}^{(k)}=\sum_{0}^{h}\left[b_{h i} J_{k-(2 i+1)}(r) \cos (k+\right. & (2 i+1)(n-1)) \varphi+ \\
& \left.+b_{h i}^{\prime} J_{k+(2 i+1)}(r) \cos (k-(2 i+1)(n-1)) \varphi\right],
\end{aligned}
$$

ove le $a, a^{\prime}, b, b^{\prime}$ sono coefficienti costanti da determinare, si ha

$$
\begin{aligned}
2 \cos (n-1) \varphi \cdot F_{2 h-1}^{(k)} & =\sum_{i}^{h-1}\left\{b_{h-1, i} J_{k-(2 i+1)}[\cos (k+(2 i+2)(n+1)) \varphi+\right. \\
+ & \cos (k+2 i(n-1)) \varphi]+b_{h-1, i}^{\prime} J_{k+(2 i+3)}[\cos (k-2 i(n-1)) \varphi+ \\
& +\cos (k-(2 i+2)(n-1)) \varphi]\},
\end{aligned}
$$

$2 \cos (n-1) \varphi \cdot F_{2 h}^{(k)}=a_{h_{0}} J_{k}[\cos (k+n-1) \varphi+\cos (k-(n-1)) \varphi]+$

$$
\begin{aligned}
& +\sum_{i}^{h}\left\{a_{n i} J_{k-2 i}[\cos (k+(2 i+1)(n-1)) \varphi+\cos (k+(2 i-1)(n-1)) \varphi]+\right. \\
& \left.+a_{h i}^{\prime} J_{k+2 i}[\cos (k-(2 i-1)(n-1)) \varphi+\cos (k-(2 i+1)(n-1)) \varphi]\right\} .
\end{aligned}
$$

Sostituendo nella prima delle $\left(6_{2}\right)$, e tenendo conto dell' equazione diffe. renziale delle funzioni di Besser, si ha

$$
\begin{aligned}
& a_{h 0}\left(\frac{4 h^{2} n^{2}}{r^{2}} J_{k}+\frac{4 h n}{r} J_{k}^{\prime}\right) \cos k \varphi+\sum_{1}^{n}\left\{4 n a _ { h i } \left[\frac{n\left(h^{2}-i^{2}\right)-i(k-2 i)}{r^{2}} J_{k-2 i}+\right.\right. \\
& \left.\left.+\frac{h}{r} J_{k-2 i}^{\prime}\right] \cos (k+2 i(n-1)) \varphi\right\}+4 n a_{h i}^{\prime}\left[\frac{n\left(h^{2}-i^{2}\right)+i(k+2 i)}{r^{2}} J_{k+2 i}+\right. \\
& \left.+\frac{h}{r} J_{k+2 i}^{\prime}\right] \cos (k-2 i(n-1)) \varphi+\frac{1}{r} \sum_{0}^{h-1}\left\{b_{h-1, i} J_{k-(2 i+1)}[\cos (k+(2 i+2)(n-1)) \varphi+\right. \\
& \left.+\cos (k+2 i(n-1)) \varphi]+b_{h-1, i}^{\prime} J_{k+(2 i+1)}[\cos (k-2 i(n-1)) \varphi+\cos (k-(2 i+2)(n-1)) \varphi]\right\}+ \\
& +\frac{1}{r^{2}} a_{h-1,0} J_{k} \cos k \varphi+\frac{1}{r^{2}} \sum_{i}^{h-1}\left[a_{h-1, i} J_{k-2 i} \cos (k+2 i(n-1)) \varphi+\right. \\
& \left.+a_{h-1, i}^{\prime} J_{k+2 i} \cos (k-2 i(n-1)) \varphi\right]=0 .
\end{aligned}
$$


Uguagliando a zero i coefficienti di $\cos k \varphi, \cos (k+2 i(n-1)) \varphi$, $\cos (k-2 i(n-1)) \varphi,(i=1.2, \ldots, h)$ si ottengono le equazioni

$$
\begin{aligned}
a_{h 0}\left(\frac{4 h^{2} n^{2}}{r^{2}} J_{k}+\frac{4 h n}{r} J_{k}^{\prime}\right)+\frac{1}{r}\left(b_{h-1,0} J_{k-1}+b_{h-1,0}^{\prime} J_{k+1}\right)+\frac{1}{r^{2}} a_{h-1,0} J_{k}=0, \\
4 n a_{h i}\left[\frac{n\left(h^{2}-i^{2}\right)-i(k-2 i)}{r^{2}} J_{k-2 i}+\frac{h}{r} J_{k-2 i}^{\prime}\right]+\frac{1}{r}\left[b_{k-1, i-1} J_{k-(2 i-1)}+b_{h-1, i} J_{k-(2 i+1)}\right]+ \\
+\frac{1}{r^{2}} a_{h-1, i} J_{k-2 i}=0, \quad(i=1,2, \ldots, h-1),
\end{aligned}
$$

$\left.\left(8_{2}\right) \quad 4 n a_{h i} \mid \frac{h(2 h-k)}{r^{2}} J_{k-2 h}+\frac{h}{r} J_{k-2 h}^{\prime}\right]+\frac{1}{r} b_{h-1, h-1} J_{k-(2 h-1)}=0$,

$$
\begin{aligned}
4 n a_{h i}^{\prime}\left[\frac{n\left(h^{2}-i^{2}\right)+i(k+2 i)}{r^{2}} J_{k+2 i}+\right. & \left.\frac{h}{r} J_{k+2 i}^{\prime}\right]+\frac{1}{r}\left[b_{h-1, i}^{\prime} J_{k+1 i+1}+b_{h-0, i-1}^{\prime} J_{k+2 i-1}\right]+ \\
& +\frac{1}{r^{2}} a_{h-1, i}^{\prime} J_{k+2 i}=0, \quad(i=1,2, \ldots, h-1),
\end{aligned}
$$

$$
4 n a_{h h}^{\prime}\left[\frac{h(2 h+k)}{r^{2}} J_{k+2 h}+\frac{h}{r} J_{k+2 h}^{\prime}\right]+\frac{1}{r} b_{h-1, h-1}^{\prime} J_{h+2 h-1}=0
$$

le quali dovranno ridursi a delle identità. Tenendo conto delle formule ricorrenti delle funzioni di Bessel la prima delle equazioni $\left(8_{2}\right)$ si può scrivere

$$
\left[4 h n(h n-k) a_{h_{0}}+a_{h-1,0}+2 k b_{h-1,0}^{\prime}\right] \frac{J_{k}}{r}+\left(4 h n a_{h_{0}}+b_{h-1,0}-b_{h-1,0}^{\prime}\right) J_{k-1}=0
$$

e avremo perciò

$$
\left\{\begin{array}{l}
4 h n(h n-k) a_{h_{0}}+a_{h-1,0}+2 k b_{h-1,0}^{\prime}=0 \\
4 h n a_{h_{0}}+b_{h-1,0}-b_{h-1,0}^{\prime}=0
\end{array}\right.
$$

Così pure la seconda delle $\left(8_{2}\right)$ diventa

$$
\begin{gathered}
\left\{4 n\left[n\left(h^{2}-i^{2}\right)-i(k-2 i)+h(k-2 i) a_{h i}+a_{h-1, i}+2(k-2 i) b_{h-1, i}\right\} \frac{J_{k-2 i}}{r}+\right. \\
+\left(b_{k-1,1-1}-b_{h-1, i}-4 n h a_{h i}\right) J_{k-2 i-1}=0,
\end{gathered}
$$

da cui segue

$$
\begin{aligned}
& 4 n\left[n\left(h^{2}-i^{2}\right)-i(k-2 i)+h(k-2 i)\right] a_{n i}+a_{n-1, i}+2(k-2 i) b_{h-1, i}=0, \\
& \left(4 n h a_{h i}+b_{h-1, i}-b_{h-1, i-1}=0, \quad(i=1,2, \ldots, h-1)\right. \text {. }
\end{aligned}
$$

Nello stesso modo dalla terza delle $\left(8_{g}\right)$ si ha

$$
-4 n h a_{h n}+b_{h-1, h-1}=0
$$

e dalle rimanenti equazioni si deduce

$$
\begin{gathered}
\left(10_{z}^{\prime}\right) \begin{cases}4 n\left[n\left(h^{2}-i^{2}\right)+i(k+2 i)-h(k+2 i)\right] a_{h i}^{\prime}+2(k+2 i) b_{h-1, i}^{\prime}+a_{h-1}^{\prime}, i=0, \\
4 n h a_{h i}^{\prime}+b_{h-1, i-1}^{\prime}-b_{h-1, i}^{\prime}=0, & (i=1,2, \ldots, h-1)\end{cases} \\
\left(11_{2}^{\prime}\right) \quad 4 n h a_{h h}^{\prime}+b_{h-1, h-1}^{\prime}=0 .
\end{gathered}
$$


Con procedimento analogo, sostituendo nella seconda delle $\left(6_{2}\right)$ in luogo di $F_{2 h+1}^{(k)}, F_{2 h}^{\mid k)}, F_{2 h-1}^{(k)}$ le espressioni che si deducono dalle $\left(7_{2}\right)$, nguagliando quindi a zero $\mathrm{i}$ coefficienti dei termini in $\cos (k+(2 i+1)(n-1)) \varphi$, e $\cos (k-(2 i+1)(n-1)) \varphi$, per $i=0,1,2, \ldots, h-1, h$, si ottengono le relazioni

$$
\begin{aligned}
& b_{h 0}\left[\frac{n^{2}\left((2 h+1)^{2}-1\right)-2 n(k-1)}{r^{2}} J_{k-1}+\frac{2 n(2 h+1)}{r} J_{k-1}^{\prime}\right]+\frac{1}{r}\left(a_{h 0} J_{k}+a_{h 1} J_{k-1}\right)+ \\
& +\frac{1}{r^{2}} b_{h-1,0} J_{k-1}=0 \\
& \left.b_{h i}\left[\frac{n^{2}\left((2 h+1)^{2}-(2 i+1)\right)+2 n(2 i+1)(2 i-k+1)}{r^{2}}\right] J_{i c-(2 i+1)}+\frac{2(2 h+1) n}{r} J_{k-(2 i+1)}^{\prime}\right]+ \\
& +\frac{1}{r}\left[a_{h i} J_{k-2 i}+a_{h, i+1} J_{k-2(i+1)}\right]+\frac{1}{r^{2}} b_{h-1, i} J_{k-(2 i+1)}=0, \quad(i=1,2, \ldots, h-1), \\
& b_{h h}\left[\frac{2 n(2 h+1)(2 h+1-k)}{r^{2}} J_{k-(2 h+1)}+\frac{2(2 h+1) n}{r} J^{\prime}{ }_{k-(2 h+1)}\right]+\frac{1}{r} a_{h h} J_{k-2 h}=0, \\
& b_{h 0}^{\prime}\left[\frac{n^{i}\left((2 h+1)^{2}-1\right)+2 n(k+1)}{r^{2}} J_{k+1}+\frac{2 n(2 h+1)}{r} J_{k+1}^{\prime}\right]+\frac{1}{r}\left(a_{h 0} J_{k}+a_{h 1}^{\prime} J_{k+2}\right)+ \\
& +\frac{1}{r^{2}} b_{h-1,0}^{\prime} J_{k+1}=0 \text {, } \\
& b^{\prime}{ }_{h i}\left[\frac{n^{2}\left((2 h+1)^{2}-(2 i+1)^{2}\right)+2 n(2 i+1)(2 i+1+k)}{r^{2}} J_{k+2 i+1}+\frac{2 n(2 h+1)}{r} J_{k+2 i+1}{ }^{2}\right]+ \\
& +\frac{1}{r}\left[a_{h i}^{\prime} J_{k+2 i}+a_{h, i+1}^{\prime} J_{k+2(i+1)}\right]+\frac{1}{r^{2}} b_{h-1, i}^{\prime} J_{k+2 i+1}=0, \quad(i=1,2, \ldots, h-1), \\
& b_{h h}^{\prime}\left[\frac{2 n(2 h+1)(2 h+1+k)}{r^{2}} J_{k+2 h+1}+\frac{2 n(2 h+1)}{r} J_{k+2 h+1}^{\prime}\right]+\frac{1}{r} a_{h h}^{\prime} J_{k+2 h}=0 .
\end{aligned}
$$

Poichè queste dorranno essere verificate identicamente, tenendo conto al solito delle formule ricorrenti fra le funzioni di Besses, si dedncono le seguenti relazioni fra $i$ coefficienti

$$
\begin{aligned}
& \left\{\begin{array}{l}
4 n h[n(h+1)+k-1] b_{h 0}+b_{h-1,0}+2(k-1) a_{h 1}=0, \\
a_{h 0}-2 n(2 h+1) b_{h 0}-a_{h 1}=0, \\
4 n(h-i)[n(h+i+1)-(2 i+1-k)] b_{h i}+b_{h-1, i}+2(k-2 i-1) a_{h, i+1}=0, \\
a_{h i}-a_{h, i+1}-2 n(2 h+1) b_{h i}=0, \\
a_{h h}-2 n(2 h+1) b_{h h}=0,
\end{array}\right. \\
& \begin{cases}4 n h[n(h+1)-(k+1)] b_{h 0}^{\prime}+b_{h-1,0}^{\prime}+2(k+1) a_{h 1}^{\prime}=0, \\
a_{h 0}-a_{h 1}^{\prime}+2 n(2 h+1) b_{h 0}^{\prime}=0, \\
4 n(h-i)[n(h+i+1)-(k+2 i+1)] b_{h i}^{\prime}+b_{h-1, i}^{\prime}+2(k+2 i+1) a_{h, i+1}^{\prime}=0, \\
a_{h i}^{\prime}-a_{h, i+1}^{\prime}+2 n(2 h+1) b_{h i}^{\prime}=0, \\
a_{h h}^{\prime}+2 n(2 h+1) b_{h h}^{\prime}=0 .\end{cases}
\end{aligned}
$$


Osserviamo ora che dalle formule che dànno $\mathscr{G}_{1}{ }^{(k)}, \mathcal{G}_{3}{ }^{(k)}$, risulta $b_{h i}^{\prime}=-b_{h i}$, e da quelle che dànno $\mathcal{E}_{i}{ }^{(k)}, \mathfrak{G}_{4}{ }^{(k)}$, si ha $a_{h i}=a_{h i}$.

Cosi facendo per $i=1,2, \ldots, h$, le equazioni $\left(9_{2}\right),\left(10_{2}\right),\left(11_{2}\right),\left(10_{2}{ }^{\prime}\right),\left(11_{2}{ }^{\prime}\right)$, $\left(12_{2}\right),\left(12_{2}\right)$, si riducono alle seguenti

$$
\begin{aligned}
& 4 h n(h n-k) a_{h 0}+a_{h-1,0}-2 k b_{h-1,0}=0, \\
& 2 h n a_{h 0}+b_{h-1,0}=0 \\
& 4 n(h-i)[n(h+i)+k-2 i] a_{h i}+a_{h-1, i}+2(k-2 i) b_{h-1, i}=0, \\
& 4 n h a_{h i}+b_{h-1, i}-b_{h-1, i-1}=0, \quad(i=1,2, \ldots, h-1) \text {, } \\
& 4 n h a_{h h}-b_{h-1, h-1}=0 \text {, } \\
& 4 n(h-i)[n(h+i)-(k+2 i)] a_{h i}+a_{h-1+i}-2(k+2 i) b_{h-1, i}=0, \\
& (i=1,2, \ldots h-1), \\
& \left\{\begin{array}{l}
4 n h[n(h+1)+k+1] b_{h 0}+b_{h-1,0}+2(k-1) a_{h 1}=0, \\
a_{h 0} \quad a_{h 1}-2 n(2 h+1) b_{h 0}=0, \\
4 n(h-i)[n(h+i+1)-(2 i+1-k)] b_{h i}+b_{h-1, i}+2(k-2 i-1) a_{h, i+1}=0, \\
a_{h i}-a_{h, i+1}-2 n(2 h+1) b_{h i}=0, \quad(i=1,2, \ldots, h-1), \\
a_{h h}-2 n\left(2 h+1, b_{h h}=0,\right. \\
4 n h[n(h+1)-(k+1)] b_{h 3}+b_{h-1,0}-2(k+1) a_{h i}=0, \\
4 n(h-i)[n(h+i+1)-(k+2 i+1)] b_{h i}+b_{h-1, i}-2(k+2 i+1) a_{h, i+1}=0, \\
\quad(i=1,2, \ldots, h-1) .
\end{array}\right.
\end{aligned}
$$

Da queste si ricava

$$
\begin{aligned}
& a_{h 0}=-\frac{b_{h-1,0}}{2 h n}, a_{h-1,0}=2 h n b_{h-1,0}, a_{h 1}=\frac{b_{h-1,0}}{2 n(h+1)}, b_{h 0}=-\frac{b_{h-1,0}}{4 h n^{2}(h+1)}, \\
& a_{h i}=\frac{b_{h-1, i-1}}{2 n(h+i)}, a_{h-1, i}=-2 n(h-i) b_{h-1, i-1}, b_{h-1, i}=-\frac{h-1}{h+1} b_{h-1, i-1},
\end{aligned}
$$

$\left(14_{2}\right) a_{h, i+1}=\frac{b_{h-1, i}}{2 n(h+i+1)}=-\frac{h-i}{2 n(h+i+1)(h+i)} b_{h-1, i-1}$,

$$
\begin{aligned}
& b_{h i}=-\frac{b_{h-1, i}}{4 n^{2}(h-i)(h+i+1)}=\frac{b_{h-1, i-1}}{4 n^{2}(h+i+1)(h+i)}, \quad(i=1,2, \ldots, h-1), \\
& a_{h h}=\frac{1}{4 n h} b_{h-1, h-1}, \quad b_{h h}=\frac{1}{8 n^{2} h(2 h+1)} b_{h-1, h-1},
\end{aligned}
$$

dalle quali segue anche

$$
\frac{a_{h, i+1}}{a_{h i}}=-\frac{h-1}{h+i+1},(i=0,1,2, \ldots, h-1) ; \quad \frac{b_{h i}}{a_{h i}}=\frac{1}{2 n(h+i+1)},(i=0,1,2, \ldots, h) .
$$


Queste formule ricorrenti permettono di esprimere i coefficienti $a_{h i}, b_{h i}$, per mezzo di $a_{k 0}$ e si ottiene

$$
\begin{aligned}
a_{h i} & =(-1)^{i} \frac{h(h-1)(h-2) \ldots(h-i+1)}{(h+1)(h+2) \ldots(h+i)} a_{h 0} \\
b_{h i} & =(-1)^{i} \frac{h(h-1)(h-2) \ldots(h-i+1)}{(h+1)(h+2) \ldots(h+i+1)} \cdot \frac{a_{h 0}}{2 n}, \quad(i=1,2 \ldots . h) \\
b_{h 0} & =\frac{a_{h 0}}{2 n(h+1)} .
\end{aligned}
$$

Dalle prime due delle $\left(14_{2}\right)$ si ha ancora

$$
a_{h 0}=-\frac{1}{4 h^{2} n^{2}} a_{h-1,0},
$$

e quindi

$$
a_{h 0}=(-1)^{h-1} \frac{a_{10}}{4^{h-1}(h !)^{2} n^{2(h-1)}}
$$

Ma dall' espressione $\left(5_{2}\right)$ della funzione $\mathcal{G}_{2}^{(k)}$ risulta che è $a_{10}=-\frac{1}{4 n^{2}}$; ne segue

$$
a_{h 0}=(-1)^{h} \frac{1}{2^{2 h} n^{2 h}(h !)^{2}}
$$

e pertanto infine

$$
\begin{aligned}
a_{h i}=\frac{(-1)^{i+h}}{(2 n)^{2 n}(h+i) !(h-i) !} ; \quad b_{h i}=\frac{(-1)^{i+h}}{(2 n)^{2 h+1}(h+i+1) !(h-i) !} \\
\quad(i=0,1,2, \ldots, h)
\end{aligned}
$$

Sostituendo nelle espressioni $\left(7_{2}\right)$ di $F_{2 h}^{(h)}, F_{2 h+1}^{(k)}$ in luogo dei coefficienti $a_{h i}, b_{h i}$ i valori ora trovati, e ricordando che $\dot{e} a_{h i}^{\prime}=a_{h i}, b_{h i}^{\prime}=-b_{h i}$, $(i=1,2, \ldots, h), b_{h 0}^{\prime}=-b_{h 0}$, e ricordando inoltre che si aveva $\mathscr{E}_{l}{ }^{(k)}=r^{j \prime} F_{j}{ }^{(k)}(r, \mathfrak{p})$, si ottiene facilmente

$$
\begin{aligned}
& \left.\mathcal{E}_{2 h}^{(k)}=\frac{(-1)^{h} r^{2 h n}}{(2 n)^{2 h}} \mid \frac{1}{(h !}\right)^{2} J_{k}(r) \cos k \varphi+\sum_{1}^{h} \frac{(-1)^{i}}{(h-i) !(h+i) !}\left[J_{k-2^{\prime}}(r) \cos (k+2 i(n-1)) \varphi+\right. \\
& \left.+J_{k+2 i}(r) \cos (k-2 i(n-1)) \varphi\right] ! \\
& \mathcal{G}_{2 h+1}^{(k)}=\frac{(-1)^{h} r^{(2 h+1) n}}{(2 n)^{2 h+1}} \sum_{0}^{h} \frac{(-1)^{i}}{(h-1) !(h+i+1) !}\left[J_{k-(2 i+1)}(r) \cos (k+(2 i+1)(n-1)) \varphi-\right. \\
& \left.-J_{k+(2 i+1)}(r) \cos (k-(2 i+1)(n-1)) \varphi\right]
\end{aligned}
$$

dalle quali seguono in particolare $\mathrm{i}$ valori di $\mathfrak{G}_{2}{ }^{(k)}, \mathfrak{G}_{3}{ }^{(k)}, \mathfrak{G}_{4}{ }^{(k)}$, già calcolati direttamente. 
5. Ritorniamo ora all'espressione $(3$,$) delle funzioni \mathcal{G}^{(k)}$, soluzioni dell'equazione differenziale $\left(2_{2}\right)$ che per $\gamma=0$ si riducono a $J_{k}(r) \cos k \varphi$, abbiamo, in virtù delle $\left(15_{z}\right)$,

$$
\begin{aligned}
& \mathcal{G}^{(k)}=J_{k}(r) \cos k \varphi+\stackrel{\Sigma}{\Sigma}_{0}^{\infty} \gamma^{2 h+1} \mathcal{G}_{2 h+1}^{(k)}+{\underset{1}{\Sigma}}_{h}^{\infty} \gamma^{2 h} \mathcal{G}_{2 h}^{(k)}= \\
& =J_{k}(r) \cos k \varphi+\sum_{0}^{\infty}(-1)^{n}\left(\frac{\gamma r^{n}}{2 n}\right)^{2 n+1}{\stackrel{\Sigma}{\Sigma_{i}}}_{0}^{n} \frac{(-1)^{i}}{(h-i) !(h+i+1) !} \cdot \\
& \cdot\left[J_{k-(2 i+1)}(r) \cos (k+(2 i+1)(n-1)) \varphi-J_{k+(z i+1)}(r) \cos (k-(2 i+1)(n-1)) \varphi\right]+ \\
& +\sum_{1}^{\infty}(-1)^{h}\left(\frac{\gamma r^{n}}{2 n}\right)^{2 h}\left\{\frac{1}{(h !)^{2}} J_{k}(r) \cos k \varphi+\sum_{i}^{h} \frac{(-1)^{t}}{(h-i) !(h+i) !} .\right. \\
& \left.\cdot\left[J_{k-2 i}(r) \cos (k+2 i(n-1)) \varphi+J_{k+z i}(r) \cos (k-2 i(n-1)) \varphi\right]\right\} \text {, }
\end{aligned}
$$

che si può scrivere

$$
\begin{aligned}
\mathcal{G}^{(k)} & =\left[1+\sum_{i}^{\infty} \frac{(-1)^{h}}{(h !)^{2}}\left(\frac{\gamma r^{n}}{2 n}\right)^{2 h}\right] J_{k}(r) \cos k \varphi+ \\
& +\sum_{i}^{\infty} \sum_{i}^{\infty} \frac{(-1)^{n+i}}{(h-i) !(h+i+1) !}\left(\frac{\gamma r^{n}}{2 n}\right)^{2 h+1}\left[J_{k-(2 i+1)} \cos (k+(2 i+1)(n-1)) \varphi-\right. \\
& +\sum_{i}^{\infty} \sum_{i}^{\infty} \frac{(-1)^{h+i}}{(h-i) !(h+i) !}\left(\frac{\gamma r^{n}}{2 n}\right)^{2 h}\left[J_{k-2 i} \cos (k \cdot 2 i(n-1)) \varphi+J_{k+2 i} \cos (k-2 i(n-1)) \varphi\right]
\end{aligned}
$$

Cambiando nelle ultime due somme $h$ in $h+i$, si ha anche

$$
\begin{aligned}
& \mathcal{G}^{(k)}=\sum_{0}^{\infty} \frac{(-1)^{h}}{(h !)^{2}}\left(\frac{\gamma r^{n}}{2 n}\right)^{2 k} \cdot J_{k}(r) \cos k \varphi+ \\
& +\sum_{0}^{\infty} \sum_{0}^{\infty} \frac{(-1)^{h}}{h !(h+2 i+1) !}\left(\frac{\gamma r^{n}}{2 n}\right)^{2(h+i)+1}\left[J_{k-(2 i+1)} \cos (k+(2 i+1)(n-1)) \varphi-\right. \\
& \left.-J_{k+(2 i+1)} \cos (k-(2 i+1)(n-1)) \varphi\right]+ \\
& +\sum_{i}^{\infty} \sum_{0}^{\infty} \frac{(-1)^{h}}{h !(h+2 i) !}\left(\frac{\gamma^{r n}}{2 n}\right)^{2(h+i)}\left[J_{k-2 i} \cos (k+2 i(n-1)) \varphi+\right. \\
& +J_{k+2 i} \cos (k-2 i(n-1) \mid \varphi]
\end{aligned}
$$

e ricordando che

risulta

$$
J_{p}(x)=\sum_{0}^{\infty} \frac{(-1)^{h}}{h !(h+p) !}\left(\frac{x}{2}\right)^{2 h+p}
$$

$$
\begin{aligned}
\mathcal{G}^{(k)}= & J_{0}\left(\frac{\gamma r^{n}}{n}\right) J_{k}(r) \cos k \varphi+ \\
& +\sum_{0}^{\infty} J_{2 i+1}\left(\frac{\gamma r^{n}}{n}\right)\left[J_{k-(2 i+1)}(r) \cos (k+(2 i+1)(n-1)) \varphi-\right. \\
& \left.\quad-J_{k+(2 i+1)}(r) \cos (k-(2 i+1)(n-1)) \varphi\right]+ \\
& +\stackrel{\Sigma}{\Sigma}_{i}^{\infty} J_{z i}\left(\frac{\gamma r^{n}}{n}\right)\left[J_{k-z i}(r) \cos (k+2 i(n-1)) \varphi+J_{k+2 i}(r) \cos (k-2 i(n-1)) \varphi\right] .
\end{aligned}
$$


Ricordando inoltre che per $p$ intero è $J_{-p}(x)=(-1)^{p} J_{p}(x)$ e quindi $J_{-2 i}=J_{2 i}, J_{-(2 i+1)}=-J_{2 i+1}$, si ha ancora

$$
\begin{aligned}
& \mathcal{G}^{(k)}=\sum_{-\infty}^{+\infty}\left[J_{k-i i}(r) J_{2 i}\left(\frac{\gamma r^{n}}{n}\right) \cos (k+2 i(n-1)) \varphi+\right. \\
& \left.+J_{k-(2 i+1)}(r) J_{2 i+1}\left(\frac{\gamma r^{n}}{n}\right) \cos (k+(2 i+1)(n-1)) \varphi\right]
\end{aligned}
$$

cioè infine

$$
\begin{array}{r}
\mathcal{G}^{(k)}=\sum_{-\infty}^{+\infty} J_{k-p}(r) \cdot J_{p}\left(\frac{\gamma r^{n}}{n}\right) \cos (k+p(n-1)) \varphi \\
k=0,1,2, \ldots
\end{array}
$$

il cui secondo membro è una serie assolutamente convergente per ogni valore di $r$, come si riconosce facilmente in base alle note espressioni delle funzioni di Bessel. Essa dà, in una forma estremamente semplice, le soluzioni dell'equazione differenziale $\left(2_{2}\right)$ delle funzioni epicicloidali che per $\gamma=0$ si riducono alle funzioni $J_{k}(r) \cos k \varphi$.

In virtù delle posizioni $\left(1_{2}\right)$ è

$$
r=\lambda n a \rho, \quad \frac{\gamma r^{n}}{n}=\lambda c a \rho^{n}
$$

ponendo allora per semplisità

$$
J_{p}^{(k)}(\rho, \lambda)=J_{k-p}(\lambda n a \rho) J_{p}\left(\lambda c a \rho^{n}\right)
$$

la $\left(16_{2}\right)$ diventa

$$
\begin{array}{r}
\mathcal{G}^{(k)}(\rho, \varphi, \lambda)={\underset{-\infty}{\Sigma_{p}}}_{-_{p}}^{+\infty} J_{p}(\rho, \lambda) \cos (k+p(n-1)) \varphi \\
k=0,1,2, \ldots
\end{array}
$$

la quale esprime le soluzioni dell' equazione differenziale $\left(4_{4}\right)$, che per $c=0$ si riducono alle $J_{k}(\lambda n a \rho) \cdot \cos k \varphi$.

6. Essendo $C_{0}, C_{1}, C_{2}, \ldots$ costanti arbitrarie abbiamo ora dell' equazione differenziale $\left(4_{1}\right)$ la soluzione più generale

$\left(10_{2}\right) \quad \mathcal{G}(\rho, \varphi, \lambda)={\underset{\Sigma}{0}}_{k}^{\infty} C_{k} \mathcal{G}^{(k)}={\underset{\Sigma}{0}}_{k}^{\infty} \underset{-\infty}{+\infty} C_{k} J_{p}^{(k)}(\rho, \lambda) \cos (k+p(n-1)) \varphi$.

Ordinandola nella forma

$$
\mathcal{G}(\rho, \varphi \lambda)=\sum_{0}^{\infty} F_{s}(\rho, \lambda) \cos s \varphi,
$$


risulta

$$
\begin{aligned}
& F_{0}(\rho, \lambda)=\sum_{0}^{\infty} C_{p(n} \quad J_{-p}^{(p(n-1))}(\rho, \lambda)=\sum_{0}^{\infty}(-1)^{p} C_{p(n-1)} J_{p n}(\lambda n a \rho) J_{p}\left(\lambda c a \rho^{n}\right), \\
& { }_{\left(20_{2}\right)} F_{q(n-1)}^{(\lambda, \rho)}=\sum_{0}^{\infty} C_{p(n-1)}\left[J_{q-p}^{(p(n-1))}(\rho, \lambda)+J_{-(q+p)}^{(p(n-1))}(\rho, \lambda)\right], \quad(q=1,2,3, \ldots), \\
& F_{q(n-1)+k}(\rho, \lambda)={\underset{D}{\Sigma}}_{0}^{\infty}\left[C_{p(n-1 \cdot+k} J_{q-p}^{(p(n-1)+k)}(\rho, \lambda)+C_{(p+1)(n-1)-k} J_{-(p+q+1)}^{(p+1)(n-1)-k)}(\rho, \lambda)\right], \\
& (q=0,1,2, \ldots ; k=1,2, \ldots, n-2) \text {, }
\end{aligned}
$$

e la $\left(19_{2}\right)$ diventa

$$
\begin{gathered}
\hat{\Theta}(\rho, \varphi, \lambda)=F_{0}(\rho, \lambda)+\sum_{1}^{\infty} F_{q(n-1)}(\rho, \lambda) \cos q(n-1) \varphi+ \\
\quad+\sum_{0}^{\infty} \sum_{1}^{n-2} F_{q(n-1)+k}(\rho, \lambda) \cos (q(n-1)+k) \varphi
\end{gathered}
$$

che si può considerare la somma delle $n-1$ funzioni epicicloidali :

$$
\begin{gathered}
E_{0}=F_{0}(\rho, \lambda)+\sum_{1}^{\infty} F_{q(n-1)}(\rho, \lambda) \cos q(n-1) \varphi, \\
E_{k}=\sum_{0}^{\infty} F_{q(n-1)+k}(\rho, \lambda) \cos (q(n-1)+k) \varphi,
\end{gathered}
$$

la prima delle quali, cioè $E_{0}$, si ottiene dalla $\left(19_{2}\right)$, annullando le costanti arbitrarie $C_{p(n-1)+k}(p=0,1,2, \ldots ; k=1,2, \ldots, n-2)$. Ciascuna delle rimanenti $E_{k}$, per un dato valore dell'indice $k$ che va da 1 ad $n-2$, si ottiene annullando tutte le costanti arbitrarie ad eccezione delle $C_{p(n-1)+k}(p=0,1,2, \ldots)$.

Possiamo scrivere dunque

$$
\mathcal{G}(\rho, \varphi, \lambda)=E_{0}(\rho, \varphi, \lambda)+\stackrel{\Sigma}{\Sigma}_{1}^{2} E_{k}(\rho, \varphi, \lambda)
$$

Osserviamo che nel caso di $n$ di.pari, $\frac{n-1}{2}$ è intero; allora per $k=\frac{n-1}{2}$, la terza delle $\left(2 \mathrm{O}_{2}\right)$ porge in particolare

$$
F_{\left(q+\frac{1}{2}\right)(n-1)}=\sum_{0}^{\infty} C_{\left(p+\frac{1}{2}\right)(n-1)}\left[J_{q-p}^{\left(\left(p+\frac{1}{2}\right)(n-1)\right)}(\rho, \lambda)+J_{-(p+q+1)}^{\left(\left(p+\frac{1}{2}\right)(n-1)\right.}(\rho, \lambda)\right] .
$$

\section{§ 3 . Le funzioni $\mathcal{E}^{*(k)}$ e soluzione generale dell' equazione differenziale delle funzioni epicicloidali.}

1. Cerchiamo ora soluzioni dell' equazione $\left(2_{2}\right)$ che per $\gamma=0$ si riducono alle $J_{k}(r)$ sen $k \varphi$, ove $J_{k}$ ò al solito la funzione di BESSEL di prima specie di ordine $k$, con $k$ intero positivo, e che indicheremo con $\mathcal{S}^{*(k)}$. Immaginando 
la $\mathcal{E}^{*(k)}$ sviluppata in serie di potenze di $\gamma$, avremo uno sviluppo del tipo

$$
\mathcal{G}^{*(k)}=J_{k}(r) \operatorname{sen} k \varphi+\Sigma_{1}^{\infty} \gamma^{j} \mathcal{G}_{j}^{*(k)} \text {. }
$$

Sostituendo questa espressione al posto di $u$ nella detta equazione differenziale, tenendo conto dell' equazione differenziale delle funzioni di Besset, e uguagliando a zero i coefficienti delle successive potenze di $\gamma$, si ottiene il sistema

$$
\begin{aligned}
& \frac{\partial^{2} \mathcal{G}_{1}{ }^{*(k)}}{\partial r^{2}}+\frac{1}{r} \frac{\partial \mathcal{G}_{1}^{*(k)}}{\partial r}+\frac{1}{r^{2}} \frac{\partial^{2} \mathcal{G}_{1}^{*(k)}}{\partial \varphi^{2}}+\mathcal{E}_{1}^{*(k)}+2 r^{n-1} J_{k}(r) \operatorname{sen} k \varphi \cdot \cos (n-1) \varphi=0, \\
& \frac{\partial^{2} \mathcal{G}_{2}^{*(k)}}{\partial r^{2}}+\frac{1}{r} \frac{\partial \mathcal{G}_{2}{ }^{*(k)}}{\partial r}+\frac{1}{r^{2}} \frac{\partial^{2} \mathcal{G}_{2}^{*(k)}}{\partial \varphi^{2}}+\mathcal{E}_{2}{ }^{*(k)}+r^{2(n-1)} J_{k}(r) \operatorname{sen} k \varphi+ \\
& +2 r^{n-1} \cos (n-1) \varphi \cdot \mathscr{G}_{1}^{*(k)}=0,
\end{aligned}
$$

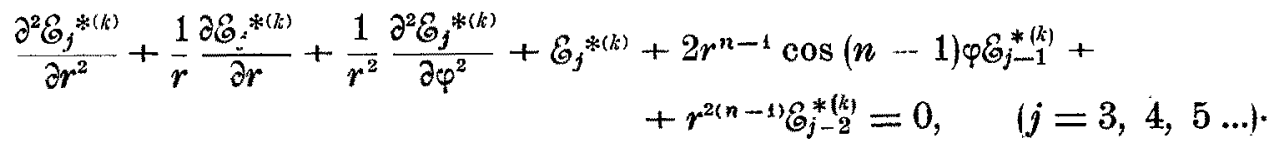

La prima di queste equazioni si può scrivere

$\frac{\partial^{2} \mathscr{G}_{1}^{*(k)}}{\partial r^{2}}+\frac{1}{r} \frac{\partial \mathcal{G}_{1}{ }^{*(k)}}{\partial r}+\frac{1}{r^{2}} \frac{\partial^{2} \mathcal{G}_{1}{ }^{*(k)}}{\partial \varphi^{-}}+\mathcal{G}_{1}^{*(k)}+r^{n-1} J_{k}(r)[\operatorname{sen}(k+n-1) \varphi+\operatorname{sen}(k-(n-1)) \varphi]=0$,

dalla quale, con calcoli analoghi a quelli con cui è stata determinata la soluzione $\mathcal{G}_{1}^{(l)}$ della prima delle equazioni $(\mathrm{I}), \S 2$, si trova la soluzione

$$
\mathcal{G}_{1}^{*(k)}=\frac{r^{n}}{2 n}\left[J_{k-1}(r) \operatorname{sen}(k+n-1) \varphi-J_{k+1}(r) \operatorname{sen}(k-(n-1)) \varphi\right] .
$$

Cosi pure dalla seconda delle (II) si ricava la soluzione $\mathcal{G}_{2}{ }^{*(k)}=\frac{r^{2 n}}{2 \cdot 4 \cdot n^{2}}\left[J_{k-2}(r) \operatorname{sen}(k+2(n-1)) \varphi-2 J_{k}(r) \operatorname{sen} k \varphi+J_{k+2}(r) \operatorname{sen}(k-2(n-1)) \varphi\right]$ e dalla terza, per $j=2 h$ ed $j=2 h+1$, si hanno le soluzioni

$$
\begin{aligned}
& \mathcal{G}_{2 h}^{*(k)}=(-1)^{h} \frac{r^{2 n h}}{(2 n)^{-h}}\left\{\frac{1}{(h !)^{2}} J_{k}(r) \text { sen } k \varphi+\sum_{i}^{h} \frac{(-1)^{i}}{(h-i) !(h+i) !} \cdot\right. \\
& \left.\cdot\left[J_{k-2 i}(r) \operatorname{sen}(k+2 i(n-1)) \varphi+J_{k+-2 i}(r) \operatorname{sen}(k-2 i(n-1)) \varphi\right]\right\}, \\
& \mathcal{E}_{2 h+1}^{*(k)}=(-1)^{n} \frac{r^{(2 h+1) n}}{(2 n)^{2 n+1}} \stackrel{n}{\Sigma}_{i}^{n} \frac{(-1)^{i}}{(h-i) !(h+i+1) !}\left[J_{k-(2 i+1)}(r) \operatorname{sen}(k+(2 i+1)) n-1, \varphi-\right. \\
& -J_{k+(2 i+1)}(r) \operatorname{sen}(k-(2 i+1)(n-1)) \varphi \text {. }
\end{aligned}
$$

I valori di queste funzioni differiscono da quelli delle corrispondenti tunzioni $\hat{G}_{j}^{(k)}$ del $\$ 2$, con lo seambio del coseno col seno. 
Sostituendo nella $\left(1_{3}\right)$ abbiamo dunque

$$
\begin{aligned}
& \mathscr{G}^{*(k)}=J_{k}(r) \operatorname{sen} k \varphi+\sum_{0}^{\infty} \gamma^{2 h+1} \mathcal{G}_{2 h+1}^{*(k)}+\sum_{1}^{\infty} \gamma^{2 h} \mathcal{G}_{2 h}^{*(k)}= \\
& =J_{k}(r) \operatorname{sen} k \varphi+\sum_{0}^{\infty}\left(-1^{n}\left(\frac{\gamma r^{n}}{2 n}\right)^{2 h+1}{\stackrel{\Sigma}{\Sigma_{i}}}_{i} \frac{(-1)^{i}}{(h-i) !(h+i+1) !} .\right. \\
& \cdot\left[J_{k-(2 i+1)}(r) \operatorname{sen}(k+(2 i+1)(n-1)) \varphi-J_{k+(2 i+1)}(r) \operatorname{sen}(k-(2 i+1)(n-1)) \varphi\right]+ \\
& +\stackrel{\Sigma}{\Sigma}_{1}^{\infty}(-1)^{n}\left(\frac{\gamma r^{n}}{2 n}\right)^{2 h}\left\{\frac{1}{(h !)^{2}} J_{k}(r) \operatorname{sen} k \varphi+\stackrel{\Sigma}{\Sigma}_{i}^{h} \frac{(-1)^{i}}{(h-i) !(h+i) !} .\right. \\
& \left.\cdot\left[J_{k-2 i}(r) \operatorname{sen}(k+2 i(n-1)) \varphi+J_{k+2 i}(r) \operatorname{sen}(k-2 i(n-1)) \varphi\right]\right\} \text {, }
\end{aligned}
$$

e con trasformazioni analoghe a quelle effettuate nel paragrafo precedente, relative alle funzioni $\mathcal{G}^{(k)}$, si ottiene

$$
\mathcal{G}^{*(k)}={\underset{-\infty}{\Sigma_{p}}}_{-\infty} J_{k-p}(r) J_{p}\left(\frac{\gamma r^{n}}{n}\right) \operatorname{sen}(k+p(n-1)) \varphi, \quad(k=0,1,2,3, \ldots)
$$

Questa, per ogni valore intero positivo o nullo di $k$, è dunque soluzione dell'equazione differenziale $\left(2_{2}\right)$ e la serie che la esprime è assolutamente convergeute per ogni valore di $r$. Essa si può scrivere anche

$$
\xi^{*(k)}(\rho, \varphi, \lambda)=\sum_{-\infty}^{+\infty} J_{p}^{(k)}(\rho, \lambda) \operatorname{sen}(k+p(n-1)) \varphi
$$

ove le $J_{p}^{(k)}(\rho, \lambda)$ sono definite dalla $\left(17_{s}\right)$.

2. Indicando con $C_{0}^{*}, C_{1}^{*}, C^{*}, \ldots$, nuove costanti arbitrarie abbiamo ora dell' equazione differenziale $\left(4_{4}\right)$ la nuova soluzione

$\left(4_{s}\right) \quad \mathcal{E}^{*}(\rho, \varphi, \lambda)=\sum_{0}^{\infty} C_{k}^{*} \mathcal{E}^{*(k)}=\sum_{0}^{\infty} C_{k}^{*} \sum_{-\infty}^{+\infty} J_{p}^{(k)}(\rho, \lambda) \operatorname{sen}(k+p(n-1)) \varphi$.

Ordinandola nella forma

$$
\mathfrak{G}^{*}(\rho, \varphi, \lambda)=\sum_{1}^{\infty} F_{s}^{*}(\rho, \lambda) \operatorname{sen} s \varphi
$$

risulta

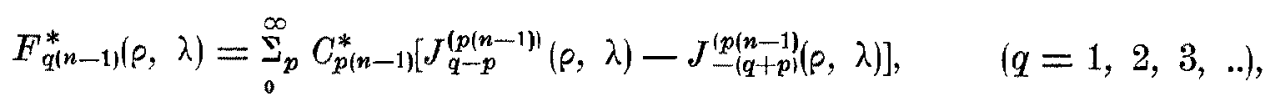

$$
\begin{array}{r}
F_{q(n-1)+k}^{*}=\stackrel{\Sigma}{0}_{p}^{\infty}\left[C_{p(n-1)+k}^{*} J_{q-p}^{p(n-1)+k)}(\rho, \lambda)-C_{(p+1)(n-1)-k}^{*} J_{-(p+q+1)}^{((p+1)(n-1)-k)}(\rho, \lambda)\right], \\
(q=0,1,3, \ldots ; k=1,2, \ldots, n-2) .
\end{array}
$$


Si può scrivere pertanto

$$
\begin{aligned}
& \mathcal{E}^{*}(\rho, \varphi, \lambda)=\sum_{1}^{\infty} F_{q(n-1)}^{*}(\rho, \lambda) \operatorname{sen} q(n-1) \varphi+ \\
& +\sum_{0}^{\infty}{ }_{1}^{n-2} \bar{\Sigma}_{k}^{2} F_{q(n-1)+k}^{*}(\rho, \lambda) \operatorname{sen}(q(n-1)+k) \varphi,
\end{aligned}
$$

e ponendo

$$
E_{0}^{*}(\rho, \varphi, \lambda)={\underset{1}{1}}_{q}^{\infty} F_{q(n-1)}^{* *}(\rho, \lambda) \operatorname{sen} q(n-1) \varphi,
$$

$\left(8_{3}\right) \quad E_{k}^{(*)}(\rho, \varphi, \lambda)=\sum_{0}^{\infty} F_{q(n-1)+k}^{*}(\rho, \lambda) \operatorname{sen}(q(n-1)+k) \varphi, \quad(k=1,2, \ldots, n-2)$, si ha anche

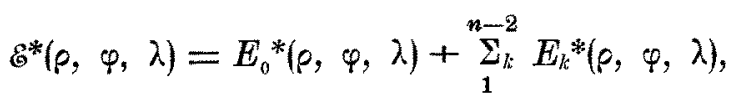

ove le $E_{0}^{*}, E_{k}^{*}$ sono ancora funzioni epicicloidali che si ottengono rispettivamente dalla $\left(4_{3}\right)$ annullando una prima volta tatte le costanti arbitrarie $C_{p(n-1)+k}^{*}(p=0,1,2, \ldots ; k=1,2, \ldots, n-2)$, e una seconda volta tutte le costanti arbitrarie ad eccezione delle $C_{p(n-1)+k}$ corrispondenti ad nn dato valore dell'indice $k$ da 1 ad $n-2$.

Osserviamo che per $n$ dispari, dando a $k$ il valore $\frac{n-1}{2}$, che e intero,

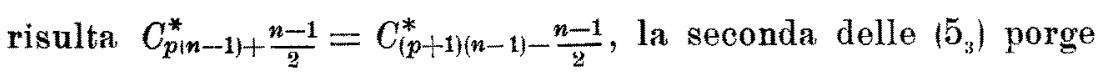

$$
F_{\left(q+\frac{1}{2}\right)(n-1)}^{*}(\rho, \lambda)=\sum_{0}^{\infty} C_{\left(p+\frac{1}{2}\right)(n-1)}^{*}\left[J_{q-p}^{\left(\left(p+\frac{1}{2}\right)(n-1)\right)}(\rho, \lambda)-J_{-(p+q+1)}^{\left(\left(p+\frac{1}{2}\right)(n-1)\right)}(\rho, \lambda)\right]
$$

e dalla $\left(8_{3}\right)$ si ha

$$
E_{\frac{n-1}{2}}^{*}(\rho, \varphi, \lambda)=\sum_{0}^{\infty} F_{\left(q+\frac{1}{2}\right)(n-1)}^{*}(\rho, \lambda) \operatorname{sen}\left(q+\frac{1}{2}\right)(n-1) \varphi
$$

3. Riunendo i risultati di questo paragrafo con quelli del paragrafo precedente, si ha che la soluzione generale dell'equazione differenziale $\left(4_{1}\right)$, regolare per $\rho=0$, sviluppata in serie di FodRIER dell' argomento $\varphi$, risulta $\left(12_{3}\right) \quad u(\rho, \varphi, \lambda)=\sum_{0}^{\infty} \underset{\Sigma_{k}}{\sum_{p}} J_{p}^{(k)}(\rho, \lambda)\left[C_{k} \cos (k+p(n-1)) \varphi+C_{k}^{*} \operatorname{sen}(k+p(n-1)) \varphi\right]$ che si può scrivere anche $\left(12^{\prime}\right) \quad u(\rho, \varphi, \lambda)=F_{0}(\rho, \lambda)+\sum_{1}^{\infty}\left[F_{q(n-1)}(\rho, \lambda) \cos q(n-1) \varphi+\right.$

$$
\begin{aligned}
& \left.+F_{q(n-1)}^{*}(\rho, \lambda) \operatorname{sen} q(n-1) \varphi\right]+\sum_{0}^{\infty} \sum_{1}^{n-2} \sum_{k}\left[F_{q(n-1)+k}(\rho, \lambda) \cos (q(n-1)+k) \varphi+\right. \\
& \left.+F_{q(n-1)+k}^{*}(\rho, \lambda) \operatorname{sen}(q(n-1)+k) \varphi\right],
\end{aligned}
$$


nella quale le funzioni $F$ ed $F^{*}$ hanno le espressioni innanzi definite. Essa si può scindere nella somma

$\left(13_{3}\right) \quad u(\rho, \varphi, \lambda)=E_{0}(\rho, \varphi, \lambda)+E_{0}^{*}(\rho, \varphi, \lambda)+\sum_{1}^{n-2}\left[E_{k}(\rho, \varphi, \lambda)+E^{*}(\rho, \varphi, \lambda)\right]$

i cui termini sono tutti funzioni epicicloidali, sono cioè soluzioni dell'equazione differenziale $\left(4_{1}\right)$.

\section{§ 4. Alcune identità notevoli.}

1. Prendiamo in considerazione la funzione epicicloidale $\mathfrak{g}^{(0)}$, che si ottiene dalla $\left(16_{2}\right)$ per $k=0$, e che si può scrivere

$\left(1_{4}\right) \quad \quad \mathcal{G}^{(0)}=J_{0}(r) J_{0}\left(\frac{\gamma r^{n}}{n}\right)+2 \sum_{1}^{\infty}(-1)^{p} J_{p}(r) J_{p}\left(\frac{\gamma r^{n}}{n}\right) \cos p(n-1) \varphi$.

Per la nota formula di NeUmanN $\left({ }^{2}\right)$

$$
J_{0}\left(\sqrt{\left.x^{2}+y^{2}-2 x y \cos \theta\right)}=J_{0}(x) J_{0}(y)+2 \sum_{1}^{\infty} J_{p}(x) J_{p}(y) \cos p \theta\right.
$$

ponendo $x=r, y=\frac{\gamma r^{n}}{n}, \theta=\pi+(n-1) \varphi$, la $\left(1_{4}\right)$ porge

$$
\mathcal{G}^{(0)}=J_{10}\left(\sqrt{r^{2}+\gamma^{2} \frac{r^{2 n}}{n^{2}}+2 \gamma \frac{r^{n+1}}{n} \cos (n-1) \varphi}\right),
$$

cioè, essendo $r=\lambda n a \rho, \gamma=c /(\lambda n a)^{n-1}$, se si pone

$$
\chi^{2}=1+2 \frac{c}{n} \rho^{n-1} \cos (n-1) \varphi+\frac{c^{2}}{n^{2}} \rho^{2(n-1)}
$$

si ha

$$
\mathcal{G}^{(0)}=J_{0}(\lambda n a \rho \chi)
$$

Ora nella mia nota citata nel $\S 1$, avevo messa la soluzione generale dell' equazione differenziale $\left(4_{1}\right)$ sotto la forma

$$
\begin{gathered}
u(\rho, \varphi, \lambda)=\frac{1}{2} A_{0} J_{0}(\lambda n a \rho \chi)+\sum_{1}^{\infty} \frac{J_{k}(\lambda n a \rho \chi)}{\chi^{k}} \sum_{0}^{k}\left(\begin{array}{l}
k \\
j
\end{array}\right)\left(\frac{c}{n}\right)^{j} \rho^{(n-1) j} . \\
\cdot\left[A_{k} \cos (k+j(n-1)) \varphi+B_{k} \operatorname{sen}(k+j(n-1)) \varphi\right]
\end{gathered}
$$

con $\dot{A}_{0}, A_{k}, B_{k}$ costanti arbitrarie, la quale per $A_{0}=2, A_{k}=B_{k}=0$, $(k=1,2,3, . .$.$) , dà proprio la \left(4_{4}\right)$.

(2) C. NeUManN, Theorie der Bessel'schen Functionen, Leipzig 1867. 
2. Per $c=0$ é $\chi=1$, e la $\left(5_{4}\right)$ porge

$$
u=\frac{1}{2} A_{0} J_{0}(\lambda n a \rho)+\sum_{1}^{\infty} J_{k}(\lambda n a \rho)\left[A_{k} \cos k \varphi+B_{k} \operatorname{sen} k \varphi\right]
$$

la quale si riduce a $J_{k}(\lambda n a \rho) \cos k \varphi=J_{k}(r) \cos k \varphi$, ponendo $A_{k}=1$ e annullando le rimanenti costanti arbitrarie

$\mathrm{Ne}$ segue che annullando nella $\left(5_{4}\right)$ tutte le costanti arbitrarie, ad eccezione di $A_{k}$, che si pone uguale ad 1 , si ha la funzione epicicloidale che abbiamo indicata con $\mathcal{G}^{(k)}$. Cioè, ricordando la $\left(16_{z}\right)$ risulta, in due modi diversi,

$$
\begin{aligned}
\mathcal{G}^{(k)} & =\frac{J_{k}(\lambda n a \rho \chi)}{\chi^{k}} \sum_{0}^{k}\left(\begin{array}{l}
k \\
j
\end{array}\right)\left(\frac{c}{n} \rho^{n-1}\right)^{j} \cos (k+j(n-1)) \varphi= \\
& =\sum_{-\infty}^{+\infty} J_{k-p}(\lambda n a \rho) J_{p}\left(\lambda c a \rho^{n}\right) \cos (k+p(n-1)) \varphi .
\end{aligned}
$$

Ponendo $\lambda n a \rho=x, \lambda c a \rho^{n}=y$, si ha la notevole identita

$$
\begin{aligned}
& \frac{J_{k}\left(\sqrt{\left.x^{2}+y^{2}+2 x y \cos (n-1) \varphi\right)}\right.}{\left[x^{2}+y^{2}+2 x y \cos (n-1) \varphi\right]^{k / 2}} \sum_{0}^{k}\left(l_{j}^{k}\right) x^{k-j} y^{j} \cos (k+j(n-1)) \varphi= \\
& =\sum_{-\infty}^{+\infty} J_{k-p}(x) \cdot J_{p}(y) \cos (k+p(n-1)) \varphi, \quad(k=0,1,2, \ldots) .
\end{aligned}
$$

Da questa, per $k=0$, si ha in particolare la formula di NEUMANN; la $(7$,$) e perciò una generalizzazione di questa formula.$

Per $\varphi=0$ la $\left(7_{4}\right)$ porge ancora

$$
J_{k}(x+y)=\sum_{-\infty}^{+\infty} J_{k-p}(x) J_{p}(y)
$$

che è una nota formula di K. NEUMANN e Schü̈FLI $\left(^{3}\right)$.

3. Dalla $\left(5_{4}\right)$ annullando tutte le costanti arbitrarie, salvo $B_{k}$, che si pone uguale ad 1 , si ottiene la funzione epicicloidale $\mathscr{G}^{*(k)}$, che per $c=0$ si riduce alla $J_{k}(\lambda n a \varphi)$ sen $k \varphi=J_{k}(r) \operatorname{sen} k \varphi$.

Ricordando allora la $\left(2_{3}\right)$, si ha la nuova relazione

$$
\begin{aligned}
& \frac{J_{k}(\lambda n a \rho \chi)}{\chi^{k}} \sum_{0}^{k}\left(\begin{array}{l}
k \\
j
\end{array}\right)\left(\frac{c}{n} \rho^{n-1}\right)^{j} \operatorname{sen}(k+j(n-1)) \varphi= \\
& =\sum_{-\infty}^{+\infty} J_{k-p}(\lambda n a \rho) J_{p}\left(\lambda c a \rho^{n}\right) \operatorname{sen}(k+p(n-1)) \varphi,
\end{aligned}
$$

ove $\chi$ ha il valore definito dalla $\left(3_{4}\right)$.

(3) Vedi per es. E. T. Whrtraker e G. N. Watson, Modern Analysis, Chap. XVII Cambridge University Press, (1946). 
Ponendo allora $\lambda n a \rho=x, \lambda c a \rho^{n}=y$, si ha l'altra identità

$$
\begin{gathered}
\frac{J_{k}\left(\sqrt{x^{2}+y^{2}+2 x y \cos (n-1)}\right)}{\left[x^{2}+y^{2}+2 x y \cos (n-1) \varphi\right]^{k / 2}} \sum_{0}^{k},\left(\begin{array}{l}
k \\
j
\end{array}\right) x^{k-j} y^{j} \text { sen }(k+j(n-1)) \varphi= \\
=\sum_{-\infty}^{+\infty} J_{k-p}(x) J_{p}(y) \operatorname{sen}(k+p(n-1)) \varphi,
\end{gathered}
$$

che è una nuova formula relativa alle funzioni di BesseL.

Essa per $k=0$ si riduce all' identità $0=0$.

4. Sommando la $\left(7_{4}\right)$ con la $\left(8_{4}\right)$ moltiplicata per l'unità immaginaria $i$, si deduce ancora la formula

$$
\begin{aligned}
& \frac{J_{k}\left(\sqrt{\left.x^{2}+y^{2}+2 x y \cos (n-1) \varphi\right)}\right.}{\left[x^{2}+y^{2}+2 x y \cos (n-1) \varphi\right]^{k / 2}} \sum_{0}^{k}\left(\begin{array}{l}
k \\
j
\end{array}\right) x^{k-j} y^{j} e^{i \cdot j(n-1) \varphi}= \\
& =\underset{-\infty}{\Sigma_{p}} J_{k-p}(x) J_{p}(y) e^{i p(n-1) \phi}, \\
& (i=\sqrt{-1})
\end{aligned}
$$

che si scinde in altre due uguagliando le parti reali e le parti immaginarie dei due membri, cioè

$$
\begin{gathered}
\left.\frac{J_{k}\left(\sqrt{x+y^{2}+2 x y \cos (n-1) \varphi}\right)}{\left(x^{2}+y^{2}+2 x y \cos (n-1) \varphi\right)^{k / 2}} \sum_{0}^{k},\left(\begin{array}{l}
k \\
j
\end{array}\right) x^{k-j} y^{j}\right\} \begin{array}{l}
\cos j(n-1) \varphi \\
\operatorname{sen} j(n-1) \varphi
\end{array}= \\
=\sum_{-\infty}^{+\infty} J_{k-p}(x) J_{p}(y)\left\{\begin{array}{l}
\cos p(n-1) \varphi \\
\operatorname{sen} p(n-1) \varphi
\end{array}\right.
\end{gathered}
$$

Moltiplicando ambo i membri della $\left(10_{4}\right)$ una volta per $\cos q(n-1) \varphi$, una seconda volta per sen $q(n-1) \varphi$, e integrando rispetto a $\varphi$ da 0 a $2 \pi$, si deducono le dae formule integrali

$$
\begin{aligned}
& J_{k-q}(x) J_{q}(y)+J_{k+q}(x) J_{-q}(y)= \\
& =\frac{1}{\pi} \int_{0}^{2 \pi} \frac{J_{k}\left(\sqrt{\left.x^{2}+y^{2}+2 x y \cos (n-1) \varphi\right)}\right.}{\left[x^{2}+y^{2}+2 x y \cos (n-1) \varphi\right]^{n / 2}} \sum_{0}^{k}\left(\begin{array}{l}
k \\
j
\end{array}\right) x^{n-j} y^{j} \cos j(n-1) \varphi \cos q(n-1) \varphi \cdot d \varphi \\
& J_{k-q}(x) J_{q}(y)-J_{k+q}(x) J_{-q}(y)= \\
& \quad=\frac{1}{\pi} \int_{0}^{2 \pi} \frac{J_{k l}\left(\sqrt{\left.x^{2}+y^{2}+2 x y \cos (n-1) \varphi\right)}\right.}{\left[x^{2}+y^{2}+2 x y \cos (n-1) \varphi\right]^{k / 2}} \sum_{0}^{k}\left(\begin{array}{l}
k \\
j
\end{array}\right) x^{k-j} y^{j} \operatorname{sen} j(n-1) \varphi \cdot \operatorname{sen} q(n-1) \varphi \cdot d \varphi,
\end{aligned}
$$

dalle quali. sommando membro a membro si ha anche

$$
\begin{gathered}
J_{k-q}(x) J_{q}(y)=\frac{1}{2 \pi} \int_{0}^{2 \pi} \frac{J_{k}\left(\sqrt{x^{2}+y^{2}+2 x y \cos (n-1) \varphi}\right)}{\left[x^{2}+y^{2}+2 x y \cos (n-1) \varphi\right]^{k / 2}} . \\
\cdot{\underset{0}{\Sigma}}_{j}^{k}\left(\begin{array}{l}
k \\
j
\end{array}\right) x^{k-j} y^{j} \cos (q-j)(n-1) \varphi \cdot d \varphi
\end{gathered}
$$


5. Osserviamo infine che facendo nella $(9),(n-1) p=\frac{\pi}{2}$, si ha in particolare l'identità

$$
\frac{J_{k l}\left(\sqrt{x^{2}+y^{2}}\right)}{\left(x^{2}+y^{2}\right)^{k / 2}}(x+i y)^{k}={\underset{-\infty}{+\infty}}_{\Sigma_{p}}^{p} J_{k-p}(x) J_{p}(y) \text {. }
$$

Ponendo in questa $x=r \cos \theta, y=r \operatorname{sen} \theta$ e uguagliando separatamente le parti reali e le parti immaginarie dei due membri, si ottengono le altre identità

$J_{k}(r) \cos k \theta=J_{k}(r \cos \theta) J_{0}(r \operatorname{sen} \theta)+\sum_{1}^{\infty}\left(-1 !^{p} J_{2 p}(r \operatorname{sen} \theta)\left[J_{k-2 p}(r \cos \theta)+J_{k+2 p}(r \cos \theta)\right]\right.$, $J_{k}(r) \operatorname{sen} k \theta=\sum_{0}^{\infty}(-1)^{p} J_{2 p+1}(r \operatorname{sen} \theta)\left[J_{k-(2 p+1)}(r \cos \theta)+J_{k+(2 p+1)}(r \cos \theta)\right]$.

\section{$\S 5.10$ Problema ai limiti.}

\section{Autosoluzioni che si annullano al contorno epicicloidale.}

1. La risoluzione del problema delle vibrazioni di una membrana epicicloidale, in base all' accennata rappresentazione conforme del campo limitato dall' epicicloide sul cerchio di raggio $\rho=1$, richiede, come si è già visto nel \$ 1, n. 2, la determinazione di autosoluzioni dell'equazione differenziale $\left(4_{1}\right)$ regolari entro il detto cerchio e che si annullano al contorno, cioè per $p=1$. Di quella equazione abbiamo la soluzione rappresentata dalla formula $\left(23_{2}\right)$ come somma delle funzioni epicicloidali $E_{0}(\rho, \varphi, \lambda), E_{k}(\rho, \varphi, \lambda),(k=1,2 \ldots$, $n-2)$, definite dalle $\left(21_{2}\right) \cdot\left(22_{2}\right)$. Affinchè questa soluzione sia nulla per $\rho=1$, e per ogui valore di $\varphi$, dovrà essere

$$
E_{0}=0, \quad E_{k}=0, \quad(k=1,2, \ldots, n-2), \quad \operatorname{per} \rho=1 .
$$

La $E_{0}=0$ per $\rho=1$, implica che sia

$$
F_{0}(1, \lambda)=0, \quad F_{q(n-1)}(1, \lambda)=0, \quad(q=1,2,3, \ldots) .
$$

Si ha così il sistema di infinite equazioni lineari omogenee nelle costanti arbitrarie $C_{p(n-1)},(p=0,1,2, \ldots)$ :

$$
\sum_{0}^{\infty} C_{p(n-1)} J_{-p}^{(p(n-1))}(1, \lambda)=0
$$

$$
{\stackrel{\infty}{\Sigma_{p}}}_{0}^{\infty} C_{p(n-1)}\left[J_{q-p}^{(p(n-1))}(1, \lambda)+J_{-(q+p)}^{(p(n-1))}(1, \lambda)\right]=0, \quad(q=1,2,3, \ldots) .
$$

La compatibilità di queste equazioni porta alla considerazione dell' equazione che si ottiene uguagliando a zero il determinante di ordine infinito dei coefficienti e che scriviamo sinteticamente cosi

$$
\left|\begin{array}{c}
J_{-p}^{(p(n-1))}(1, \lambda) \\
J_{q-p}^{(p(n-1))}(1, \lambda)+J_{(q+p)}^{(p(n-1))}(1, \lambda)
\end{array}\right|=0,
$$

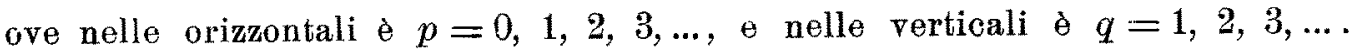


Indicando con $\lambda_{0}{ }^{(s)},(s=1,2,3, \ldots)$, le radici positive di questa equazione in $\lambda$, ordinate secondo valori crescenti, $\theta$ con $D_{p(n-1)}^{(s)}$, i complementi algebrici degli elementi della prima riga calcolati per $\lambda=\lambda_{0}{ }^{(s)}$, possiamo assumere, in correlazione a ciascun autovalore $\lambda_{0}{ }^{(s)}$ del parametro $\lambda$,

$$
C_{p(n-1)}=D_{p(n-1)}^{(s)}, \quad(p=0,1.2,3, \ldots) .
$$

Si hanno così dalle $(20$, , a meno di uno stesso fattore costante, le corrispondenti autofunzioni

$$
\begin{aligned}
& F_{0}^{(s)}\left(\rho, \lambda_{0}{ }^{(s)}\right)=\sum_{0}^{\infty} D_{p(n-1)}^{(s)} J_{-p}^{(p(n-1))}\left(\rho, \lambda_{0}^{(s)}\right), \\
& F_{q(n-1)}^{(s)}\left(\rho, \lambda_{0}^{(s)}\right)=\sum_{0}^{\infty} D_{p(n-1)}^{(s)}\left[J_{q-p}^{(p(n-1))}\left(\rho, \lambda_{0}^{(s)}\right)+J_{-(q+p)}^{(p \mid n-1))}\left(\rho, \lambda_{0}^{(s)}\right], \quad(q=1,2,3, \ldots)\right.
\end{aligned}
$$

e le autosoluzioni

$$
E_{0}^{(s)}=F_{0}^{(s)}\left(\rho, \lambda_{0}^{(s)}\right)+\sum_{1}^{\infty} F_{q(n-1)}^{(s)}\left(\rho, \lambda_{0}{ }^{(s)}\right) \cdot \cos q(n-1) \varphi .
$$

2. Consideriamo ora le condizioni $E_{k}=0,(k=1,2, \ldots, n-2)$, per $\rho=1$; ciascuna di queste dà luogo alle equazioni

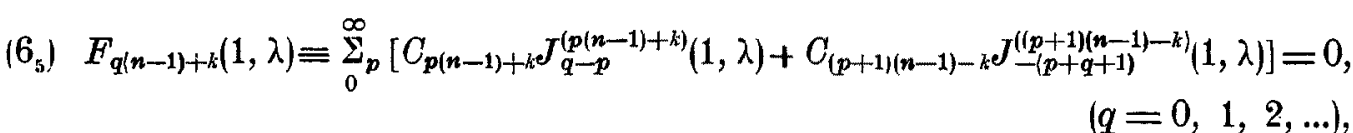

che, per un dato valore dell'indice $k$, che va da 1 ad $n-2$. costituiscono un sistema di infinite equazioni lineari omogenee nelle costanti $C_{p_{(n-1)+k}}$, $(p=0,1.2, \ldots)$. Per la loro compatibilita sarà nullo il determinante di ordine infinito formato coi coefficienti. Si ha così, per la determinazione di nuovi autovalori del parametro $\lambda$, l'equazione, scritta sotto forma simbolica

$$
\left|J_{q-p}^{(p(n-1)+k)}(1, \lambda), \quad J_{-(p+q+1)}^{((p+1)(n-1)-k)}(1, \lambda)\right| \mid=0, \quad(k=1,2, \ldots, n-2),
$$

ove nelle orizzontali $े$

Indicando con $\lambda_{k}^{(s)},(s=1,2,3, \ldots)$, le radici positive dell' equazione $\left(7_{5}\right)$, ordinate secondo valori crescenti, e con $D_{p(n-1)+k}^{(s)}, D_{(p+1)(n-1)-k}^{(s)}$, i complementi algebrini degli elementi della prima riga di ciascuno dei determinanti $\left(7_{5}\right)$, calcolati per $\lambda=\lambda_{k}^{(s)}$, possiamo porre, per un dato autovalore $\lambda_{k}^{(s)}$

$$
C_{p(n-1)+k}=D_{p(n-1)+k}^{(s)}, \quad C_{(p+1)(n-1)-k}=D_{(p+1)(n-1)-k}^{(s)}:
$$

Avremo quindi le corrispondenti antofunzioni

$$
\begin{gathered}
F_{q(n-1)+k}^{(s)}\left(\rho, \lambda_{k}^{(s)}\right)=\sum_{0}^{\infty}\left[D_{p(n-1++k}^{(s)} J_{q-p}^{(p(n-1)+k}\left(\rho, \lambda_{k}^{(s)}\right)+\uparrow\right. \\
\left.+D_{(p+1)(n-1)-k} J_{-(p+q+1)}^{(p+1)(n-1)-k)}\left(\rho, \lambda^{(s)}\right)\right]
\end{gathered}
$$


e le autosoluzioni

$$
\begin{aligned}
E_{k}^{(s)}\left(\rho, \varphi, \lambda_{k}^{(s)}\right)=\sum_{\theta}^{\infty} F_{q(n-1)+k}^{(s)}\left(\rho, \lambda_{k}^{(s)}\right) \cos (q(n-1)+k) \varphi & \\
& (k=1,2, \ldots, n-2: s=1,2,3, \ldots) .
\end{aligned}
$$

Osserviamo che nel caso di $n$ dispari, per $k=\frac{n-1}{2}$, risulta

$$
C_{p(n-1)+k} \equiv C_{(p+1)(n-1)-k}=C_{\left(p+\frac{1}{2}\right)(n-1)} ;
$$

le equazioni $\left(6_{5}\right)$ diventano allora

$$
\begin{aligned}
& F_{\left(q+\frac{1}{2}\right)(n-1)}(1, \lambda)={\stackrel{\infty}{\Sigma_{p}}}_{p} C_{\left(p+\frac{1}{2}\right)(n-1)}\left[\underset{q-p}{J\left(\left(p+\frac{1}{2}\right)(n-1)\right)}(1, \lambda)+\underset{-(p+q+1)}{J\left(\left(p+\frac{1}{2}\right)(n-1)\right)}(1, \lambda)\right]=0, \\
& (q=0,1,2, \ldots)
\end{aligned}
$$

e la corrispondente equazione determinante risulta

$$
\left.\| \underset{q-p}{J\left(\left(p+\frac{1}{2}\right)(n-1)\right.}\right)_{(1, \lambda)+J_{-(p+q+1)}\left(\left(p+\frac{1}{2}\right)(n-1)\right.}(1, \lambda) \|=0, \quad\left(\begin{array}{l}
p=0,1,2, \ldots \text { nelle orizzontali } \\
q=0,1,2, \ldots \text { nelle verticali }
\end{array}\right) .
$$

Indicando con $\frac{\lambda_{n-1}^{(s)}}{2}$ le radici positive di questa equazione (in ordine crescente) e eon $D_{\left(p+\frac{1}{2}\right)(n-1)}^{(s)}$ i complementi algebrici degli elementi della prima riga del determinante, calcolati per $\lambda=\frac{\lambda_{\frac{n}{2}-1}^{(s)}}{2}$, si può porre in questo caso

$$
C_{\left(p+\frac{1}{2}\right)(n-1)}=D_{\left(p+\frac{1}{2}\right)(n-1)}^{(s)} .
$$

Si hanno quindi le corrispondenti autofunzioni

$$
\left.F_{\left(q+\frac{1}{2}\right)(n-1)}^{(s)}\left(\rho, \lambda_{\frac{n-1}{2}}^{(s)}\right)=\sum_{0}^{\infty} D_{\left(p+\frac{1}{2}\right)(n-1)}\left[J_{q-p}^{\left(\left(p+\frac{1}{2}\right)(n-1)\right)}\left(\rho, \lambda_{\frac{n-1}{2}}^{(s)}\right)+J_{-(p+q+1)}^{\left(\left(p+\frac{1}{2}\right)(n-1)\right.}\right)\left(\rho, \frac{\left.\lambda_{\frac{n-1}{2}}^{(s)}\right)}{2}\right)\right]
$$

e le autosoluzioni

$$
E_{\frac{n-1}{2}}^{(s)}=\sum_{0}^{\infty} F_{\left(q+\frac{1}{2} /(n-1)\right.}^{(s)}\left(\rho, \frac{\lambda_{n-1}^{(s)}}{2}\right) \cos \left(q+\frac{1}{2}\right)(n-1) \varphi,
$$

3. Le autosoluzioni $E_{k}^{(s)}\left(\rho, \varphi, \lambda_{k}^{(s)}\right),(k=0.1,2, \ldots, n-2 ; s=1.2,3, \ldots)$, formano un sistema ortogonale; cioè per due valori distinti qualsiasi $k$ e $k^{\prime}$ dell' indice $k$, che va da 0 ad $n .2$, oppure per due valori distinti $s, s^{\prime}$ del. l'indice $s$, si ha la relazione di ortogonalità

$$
\int_{i}^{2 \pi} d \varphi \int_{0}^{1}\left[1+2 c \rho^{n-1} \cos (n-1) \varphi+c^{2} \rho^{2(n-1)}\right] E_{k}^{(s)} \cdot E_{k^{\prime}}^{\left(s^{\prime}\right)} \rho d \rho=0
$$

$$
\left(k \neq k^{\prime} \text { ed } s, s^{\prime} \text { qualsiasi; oppure } s \neq s^{\prime} \text { e } k, k^{\prime}\right. \text { qualsiasi). }
$$


Invero, dall' equazione differenziale $\left(4_{1}\right)$ a cui soddisfano le funzioni $E_{k}^{(s)}$, $E_{k^{\prime}}\left(s^{\prime}\right)$, segue

$$
\begin{aligned}
& \frac{\partial}{\partial \rho}\left\{\rho\left(E_{k}^{(s)} \frac{\partial E_{k^{\prime}}^{\left(s^{\prime}\right)}}{\partial \rho}-E_{k^{\prime}}^{\left(s^{\prime}\right)} \frac{\partial E_{k}^{(s)}}{\partial \rho}\right)\right\}+\frac{1}{\rho} \frac{\partial}{\partial \varphi}\left(E_{k}^{(s)} \frac{\partial E_{k^{\prime}}{ }^{(s)}}{\partial \varphi}-E_{k^{\prime}}{ }^{(s)} \frac{\partial E_{k}^{(s)}}{\partial \varphi}\right)+ \\
& +n^{2} a^{2}\left(\lambda_{k}^{(s)}-\lambda_{k^{\prime}}\left(s^{\prime}\right)\left[1+2 c \rho^{n-1} \cos (n-1) \varphi+c^{2} \rho^{2(n}{ }^{1)}\right] \rho E_{k}{ }^{(s)} E_{k^{\prime}}{ }^{\left(s^{\prime}\right)}=0\right. \text {. }
\end{aligned}
$$

Integrando ambo i membri di questa equazione rispetto a $\rho$ da 0 ad 1 , rispetto a $\varphi$ da 0 a $2 \pi$, e osservando che

$$
\left(E_{k}^{(s)} \frac{\partial E_{k^{\prime}}\left(s^{\prime}\right)}{\partial \varphi}-E_{k^{\prime}}{ }^{\left(s^{\prime}\right)} \frac{\partial E_{k}^{(s)}}{\partial \varphi}\right)_{0}^{2 \pi}=0, \quad E_{k}^{(s)}=E_{k^{\prime}}^{\left(s^{\prime}\right)}=0, \quad \text { per } \rho=1,
$$

si deduce immediatamente la $\left(10_{5}\right)$ per $k \neq k^{\prime}$, oppure per $s \neq s^{\prime}$.

4. Dell' equazione differenziale $\left(4_{i}\right)$ delle funzioni epicicloidali abbiamo ottenuta ancora nel $\$ 3$ la soluzione $\mathcal{G}^{*}(\rho, \varphi, \lambda)$, che si scinde anch' essa nella somma delle funzioni epicicloidali $E_{0}^{*}, E_{k}{ }^{*},(k=1,2, \ldots, n-2)$, definite dalle $\left(7_{3}\right),\left(8_{3}\right)$.

Affinchè sia $\mathscr{G}^{*}=0, p^{\varkappa} \rho=1$, deve essere separatamente

$$
E_{0}^{*}=0, \quad E_{k}^{*}=0, \quad(k=1,2, \ldots, n-2), \quad \text { per } \rho=1 .
$$

La $E_{0}^{*}=0$. per $\rho=1$, richiede che sia

$$
F_{q(n-1)}^{*}(1, \lambda)=\sum_{0}^{\infty} C_{p(-1)}^{*}\left[J_{q-p}^{(p(n-1))}(1, \lambda)-J_{-(p+q)}^{(p(n-1))}(1, \lambda)\right]=0, \quad(q=1,2,3, \ldots),
$$

dalle quali segue l'equazione determinante

$$
\left\|J_{q-p}^{(p(n-1))}(1, \lambda)-J_{-(p+q)}^{(p / n-1)}(1, \lambda)\right\|=0
$$

le cui radici positive $\lambda_{0}^{*(s)}$, ordinate secondo $i$ valori crescenti, forniscono $i$ corrispondenti autovalori del parametro $\lambda$. Indicando con $D_{p(n-1)}^{*(s)}$ i complementi algebrici degli elementi della prima riga di questo determinante si può porre $O_{p(n-1)}^{*}=D_{p(n-1)}^{*(s)}$. Si hanno così le autofunzioni

$$
F_{q(n-1)}^{*}\left(\rho, \lambda_{0}^{*(s)}\right)=\sum_{0}^{\infty} D_{p(n-1)}^{*(s)}\left[J_{q-p}^{(p(n-1)}\left(\rho, \lambda_{0}^{*(s)}\right)-J_{-(p+q)}^{(p(n-1))}\left(\rho, \lambda_{1}^{*(s)}\right)\right]
$$

e le autosoluzioni

$$
(q=1,2,3, \ldots)
$$

$$
E_{0}^{*(s)}\left(\rho, \varphi, \lambda_{0}^{*(s)}\right)=\sum_{1}^{\infty} F_{q(n-1)}^{*}\left(\rho, \lambda_{0}^{(s)}\right) \text { sen } q(n-1) \varphi .
$$

Passando alle condizioni $E_{k}{ }^{*}=0$, per $\rho=1,(k=1,2 . \ldots, n-2)$, in virtù delle $\left(8_{3}\right)$ e $\left(6_{3}\right)$ dovremo avere

$$
\begin{aligned}
\stackrel{\infty}{\Sigma}_{p}\left[C_{p(n-1)+k}^{*} J_{q-p}^{(p(n-1)+k)}(1, \lambda)-C_{(p+1)(n-1)-k}^{*} J_{-(p+p+1)}^{(p+k)(n-1)-k)}(1, \lambda)\right]=0, \\
(q=0,1,2,3, \ldots ; k=1,2, \ldots, n-2) .
\end{aligned}
$$


Considerando come incognite le costanti $C_{p(n-1)+k}^{*},-C_{(p+1(n-1)-k}^{*}$, l'equazione determinante degli antovalori del parametro $\lambda$, per ogni $k$ da 1 ad $n-2$, risulta

$\left\|J_{q-p}^{(p(n-1)+k)}(1, \lambda), \quad J_{-(p+q+1)}^{((p+1)(n-1)-k)}(1, \lambda)\right\|=0, \quad\left(\begin{array}{l}p=0,1,2,3, \ldots \text { nelle orizzontali } \\ q=0,1,2,3, \ldots \text { nelle verticali }\end{array}\right)$.

Ma questa non d̀ altro che l'equazione $\left(7_{5}\right)$, le cui radici positive abbiamo indicato con $\lambda_{k}^{(s)}$, possiamo percio porre

$$
C_{p(n-1)+k}^{*}=D_{p(n-1)+k}^{(s)}, \quad-C_{(p+1)(n-1)-k}^{*}=D_{(p+1)(n-1)-k}^{(s)} .
$$

Per la seconda delle $\left(5_{3}\right)$ si hanno allora le autofunzioni

$$
\begin{aligned}
& F_{q(n-1)+k}^{*(s)}\left(\rho, \lambda_{k}(s)\right)=\sum_{0}^{\infty}\left[D_{p(n-1)+k}^{(s)} J_{q-p}^{(p(n-1)+k)}\left(\rho, \lambda_{k}{ }^{(s)}\right)+\right. \\
& \left.+D_{(p+1)(n-1)-k}^{(s)} J_{-(p+q+1)}^{((p+1)(n-1)-k)}\left(\rho, \lambda_{k}(s)\right)\right], \quad(q=0,1,2, \ldots ; k=1,2, \ldots, n-2)
\end{aligned}
$$

cioè, ricordando la $\left(8_{5}\right)$ risulta

$\left(14^{\prime}{ }_{5}\right) \quad F_{q(n-1)+k}^{*(s)}=F_{q(n-1)+k}^{(s)}, \quad(q=0,1,2, \ldots ; k=1,2, \ldots, n-2 ; s=1,2,3, \ldots)$.

Le corrispondenti autosoluzioni sono:

$$
\begin{aligned}
E_{k}^{*(s)}\left(\rho, \varphi, \lambda_{k}{ }^{(s)}\right)= & \sum_{0}^{\infty} F_{q(n-1)+k}^{(s)}\left(\rho, \lambda_{k}^{(s)}\right) \operatorname{sen}(q(n-1)+k) \varphi \\
& (k=1,2, \ldots, n-2 ; s=1,2,3, \ldots) .
\end{aligned}
$$

Nel caso di $n$ dispari occorre considerare separatamente la condizione

$$
E_{\frac{n-1}{2}}^{*}=0, \quad \text { per } \rho=1,
$$

la quale in virtù delle $\left(11_{3}\right)$ e $\left(10_{3}\right)$ richiede che sia

$$
\begin{array}{r}
F_{\left(q+\frac{1}{2}\right)(n-1)}^{*}(1, \lambda)=\sum_{0}^{\infty} C_{\left(p+\frac{1}{2}\right)(n-1)}^{*}\left[\underset{q-p}{\left(\left(p+\frac{1}{2}\right)(n-1)\right)}(1, \lambda)-\underset{-(p+q+1)}{J\left(\left(p+\frac{1}{2}\right)(n-1)\right)}(1, \lambda)\right]=0 \\
(q=0,1,2, \ldots) .
\end{array}
$$

Ne segue l'equazione determinante

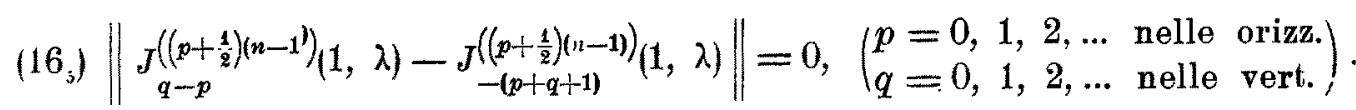

Dette $\frac{\lambda_{n-1}^{*}}{2(s)}$ le radici positive di essa (ordinate secondo i valori crescenti), se si pone

$$
C_{\left(p+\frac{1}{2}\right)(n-1)}^{*}=D_{\left(p+\frac{1}{z}\right)(n-1)}^{*(s)}, \quad(p=0,1,2, \ldots)
$$


ove i secondi membri indicano i complementi algebrici degli elementi della prima riga del determinante $\left(16_{5}\right)$, calcolati per $\lambda=\frac{\lambda_{\frac{n-1}{2}}^{*(s)}}{\text {, }}$, si hanno le auto-
funzioni

$$
\begin{aligned}
& F_{\left(q+\frac{1}{2}\right)(n-1)}^{*(\rho)}\left(\rho, \frac{\lambda_{n-1}^{*(s)}}{2}\right)=\sum_{0}^{\infty} D_{\left(p+\frac{1}{2}\right)(n-1)}^{*(s)}\left[J_{q-p}^{\left(\left(p+\frac{1}{2}\right)(n-1)\right)}\left(\rho, \frac{\lambda_{n-1}^{*(s)}}{2}\right)-\right. \\
& \left.-J_{-(p+q+1)}^{\left(\left(p+\frac{1}{2}\right)(n-1)\right.}\left(\rho, \frac{\lambda_{n-1}^{*(8)}}{2}\right)\right], \quad(q=0,1,2, \ldots),
\end{aligned}
$$

e le antosoluzioni

$$
E_{\frac{n-1}{2}}^{*(s)}=\stackrel{\infty}{\Sigma}_{\theta}^{\infty} F_{\left(q+\frac{1}{2}\right)(n-1)}^{*(s)}\left(\rho, \frac{\lambda_{n-1}^{*(s)}}{2}\right) \operatorname{sen}\left(q+\frac{1}{2}\right)(n-1) \varphi
$$

Si riconosce facilmente anche qui che le antosoluzioni $E_{0}^{*(s)}, E_{k}^{*(s)}$, $(k=1,2, \ldots, n-2)$ soddisfano alla relazione $\left(10_{5}\right)$ di ortogonalità, o meglio che l'insieme di autosoluzioni $E_{0}^{(s)}, E_{k}^{(s)}, E_{0}{ }^{*(s)}, E_{k}{ }^{*(s)}$, dell'equazione differensiale $\left(4_{1}\right)$, che si annullano per $\rho=1$, e precedentemente caratterizzate, formano un sistema fondamentale di autosoluzioni, a due a due soddisfacenti alla relazione di ortogonalità $\left(10_{5}\right)$.

5 Riunendo i risultati di questo paragrafo, la soluzione generale dell'equazione differenziale $\left(4_{1}\right)$ delle funzioni epicicloidali, regolare per $\rho=0$, e che si annulla per $p=1$, risulta :

Per $n$ pari

$\left(19_{5}\right) \quad u(\rho, \varphi)=\Sigma_{1}\left\{M_{0}^{(s)}\left[F_{0}{ }^{(s)}\left(\rho, \lambda_{0}{ }^{(s)}\right)+\sum_{1}^{\infty} F_{q(n-1)}^{(s)}\left(\rho, \lambda_{0}^{(s)}\right) \cos q(n-1) \varphi\right]+\right.$

$$
+N_{0}^{(s)} \sum_{1}^{\infty} F_{q(n-1)}^{*(s)}\left(\rho, \lambda_{0}^{*(s)}\right) \text { sen } q(n-1) \varphi+
$$

$\left.+\stackrel{\Sigma}{\Sigma}_{1}^{n-2}{\underset{0}{\Sigma_{q}}}_{q}^{\infty} F_{q(n-1)+k}^{(s)}\left(\rho, \lambda_{k}^{(s)}\right)\left[M_{k}^{(s)} \cos (q(n-1)+k) \varphi+N_{k}^{(s)} \operatorname{sen}(q(n-1)+k) \varphi\right]\right\}$, ove le $M_{n}^{(s)}, N_{0}^{(s)}, M_{k}^{(s)}, N_{k}^{(s)}$ sono costanti arbitrarie ed ove le autofunzioni $F$, $F^{*}$ sono quelle precedentemente definite

Per $n$ dispari la ${ }_{1}^{n-2}$ contiene il termine intermedio corrispondente a $k=\frac{n-1}{2}$, e che vale

$$
\begin{aligned}
& \Sigma_{1}{ }_{0} \sum_{q}^{\infty}\left[M_{\frac{n-1}{2}}^{(s)} F_{\left(q+\frac{1}{2}\right)(n-1)}^{(s)}\left(\rho, \frac{\lambda_{n-1}^{(s)}}{2}\right) \cos \left(q+\frac{1}{2}\right)(n-1) \varphi+\right. \\
& \left.\quad+N_{\frac{n-1}{2} F_{\left(q+\frac{1}{2}\right)(n-1)}^{(s)}}^{*(s)}\left(\rho, \lambda_{\frac{n-1}{2}}^{*(s)}\right) \operatorname{sen}\left(q+\frac{1}{2}\right)(n-1) \varphi\right] .
\end{aligned}
$$

con $M_{\frac{n-1}{2}}^{(s)}$ ed $N_{\frac{n-1}{2}}^{(s)}$ ancora costanti arbitrarie. 
6. Sinteticamente la $\left(19_{5}\right)$ si può scrivere:

$$
u(\rho, \varphi)=\Sigma_{1}\left\{M_{0}^{(s)} E_{0}^{(s)}+N_{0}^{(s)} E_{0}{ }^{*(s)}+\sum_{1}^{n-2}\left[M_{k}^{(s)} E_{k}^{(s)}+N_{k}^{(s)} E_{k}^{*(s)}\right]\right\}
$$

e questa permette di risolvere il problema delle vibrazioni di una membrana con contorno epicicloidale fisso, quando sia assegnato lo spostamento di ogni punto della membrana all' istante iniziale,

Il problema equivale analiticamente a determinare i coefficienti costanti $M_{0}^{(s)}, N_{0}^{(s)}, M_{k}{ }^{(s)}, N_{k}^{(s)}$ della $\left(19_{5}{ }^{\prime}\right)$ nell'ipotesi che sia assegnato il valore della funzione $u(p, \varphi)$ nell' interno del campo epicicloidale e che questa ammetta uno sviluppo del tipo $\left(19_{5}\right)$.

Tenendo conto delle relazioni di ortogonalita fra le funzioni $E_{0}{ }^{(s)}, E_{0}{ }^{*(s)}$, $E_{k}^{(s)}, E_{k}^{*(s)}$, si ricava dalla stessa $\left(19_{5}^{\prime}\right)$ :

$$
\begin{aligned}
& M_{k}(s) \int_{0}^{2 \pi} d \varphi \int_{0}^{1}\left[1+2 c \rho^{n-1}\right.\left.\cos (n-1) \varphi+c^{2} \rho^{2(n-1)}\right]\left(E_{k}^{(s)}\right)^{2} \rho d \rho= \\
&=\int_{0}^{2 \pi} d \varphi \int_{0}^{1}\left[1+2 c \rho^{n-1} \cos (n-1) \varphi+c^{2} \rho^{2(n-1)}\right] u(\rho, \varphi) E_{k}^{(s)} \cdot \rho d \rho,
\end{aligned}
$$

$$
\begin{gathered}
N_{k}^{(s)} \int_{0}^{2 \pi} d \varphi \int_{0}^{1}\left[1+2 c \rho^{n-1} \cos (n-1) \varphi+c^{2} \rho^{2(n-1)}\right]\left(E_{k}^{*(s)}\right)^{2} \rho d \rho= \\
=\int_{0}^{2 \pi} d \varphi \int_{0}^{1}\left[1+2 c \rho^{n-1} \cos (n-1) \varphi+c^{2} \rho^{2(n-1)}\right] u\left(\rho, \varphi \mid E_{k}^{*(s)} \cdot \rho d \rho\right. \\
(k=0,1,2, \ldots, n-1 ; s=1,2,3, \ldots),
\end{gathered}
$$

le quali forniscono i valori delle costanti $M_{k}^{(s)}, N_{k}^{(s)}$ per ogni $k$ da 0 ad $n-2$ e per tutti i valori dell'indice $s$.

$\grave{\mathrm{E}}$ ovvio che gli autovalori del parametro $\lambda$ definiscono le frequenze proprie di vibrazione della membrana epicicloidale considerata.

7. Considerando ancora il problema della propagazione guidata di onde elettromagnetiche in un tubo cilindrico epicicloidale indefinito, riempito di dielettrico omogeneo, nel caso di onde di tipo elettrico, che sono state caratterizzate nel $\S 1$, n. $3,2^{\circ}$. la componente $\mathcal{E}_{z}$ del campo elettrico, che deve verificare la $\left(4_{4}\right)$ e annullarsi per $\rho=1$, si può identificare, a meno di un fattore costante dipendente dalle unità di misura, con una qualsiasi delle autosoluzioni $E_{0}^{(s)}, E_{0}^{*(s)}, E_{k}^{(s)}, E_{k}^{*(s)},(k=1,2, . ., n-2 ; s=1,2,3, \ldots)$. 
Ciascuno degli autovalori del parametro $\lambda$, ricordando che era $\lambda^{2}=k^{2} \varepsilon \mu-\alpha^{2}$, stabilisce per la corrispondente autosoluzione, una relazione fra la costante di frequenza $k$ e la velocità di propagazione $V=\frac{k c_{0}}{\alpha}$ secondo l'asse del tubo.

8. In questo paragrato, per la determinazione degli autovalori del parametro $\lambda$, siamo stati condotti a considerare delle equazioni in $\lambda$ in ciascuna delle quali il primo membro è un determinante di ordine infinito.

La loro considerazione sarà legittima se essi risultano convergenti, se cioè (") converge assolutamente il prodotto degli elementi della diagonale principale ed inoltre converge assolutamente la somma degli elementi non diagonali. In tal caso, nei calcoli concreti, fissando un certo ordine di approssimazione, basterà limitarsi a considerare un determinante di ordine finito formato con gli elementi di un opportuno numero di prime righe e di prime colonne.

Riferiamoci per esempio al determinante che è a primo membro della $\left(2_{5}\right)$. il quale, quando si moltiplicano tutti gli elementi della prima riga per 2 , sí può scrivere anche

$\left(20_{6}\right) \quad\left\|J_{q-p}^{(p(n-1))}(1, \lambda)+J_{-(p+q)}^{(p(n-1)}(1, \lambda)\right\|=0, \quad\left(\begin{array}{ll}p=0,1,2, \ldots & \text { nelle orizzontali } \\ q=0,1,2, . . & \text { nelle verticali }\end{array}\right)$,

e facciamo vedere che esso è convergente.

Ricordiamo intanto la formula

$$
J_{k}(\tau)=\frac{\tau^{k}}{1 \cdot 3 \ldots(2 n-1) \pi} \int_{0}^{\pi} \cos (\tau \cos \theta) \operatorname{sen}^{2 \kappa} \theta d \theta
$$

dalla quale si deduce

ciò̀

$$
\left|\frac{J_{k}(\tau)}{\tau^{k}}\right|<\frac{1}{1.3 \ldots(2 n-1) \pi} \int_{0}^{\pi} \operatorname{sen}^{2 k} \theta d \theta
$$

$$
\left|\frac{J_{k}(\tau)}{\tau^{k}}\right|<\frac{1}{2^{k} \cdot k !}
$$

Posto allora ber brevità $\tau=\sqrt{x^{2}+y^{2}+2 x y \cos (n-1) \varphi}$, dall'identità (11, supposto $x, y$, entrambi positivi, si ottiene

$$
\begin{gathered}
\left|J_{k-p, p}(x) \cdot J_{p}(y)\right|<\frac{1}{2 \pi} \sum_{\| t}^{k}\left(\begin{array}{l}
k \\
j
\end{array}\right) x^{k-j} y^{j} \int_{0}^{2 \pi}\left|\frac{J_{k}(\tau)}{\tau^{k}}\right| \cdot|\cos (p-j)(n-1) \varphi| d \varphi< \\
<\sum_{0}^{k}\left(\begin{array}{l}
k \\
j
\end{array}\right) x^{k-j} y^{j} \cdot \frac{1}{2^{k} \cdot k !},
\end{gathered}
$$

(4) Vedi E. T. Whittaker e G. N. Watson, loco citato, Chap. IT, 28. 
ovvero

$$
\mid J_{k-p}(x) \cdot J_{p}(y)<\frac{(x+y)^{k}}{2^{k} \cdot k !}
$$

Per $x=\lambda n a, y=\lambda c a, x+y=\lambda n a\left(1+\frac{c}{n}\right)$, risulta pertanto

$$
\left|J_{p}^{(k)}(1, \lambda)\right|=\left|J_{k-p}(\lambda n a) \cdot J_{p}(\lambda c a)\right|<\frac{(\lambda n a)^{k}}{2^{k} k !}\left(1+\frac{c}{n}\right)^{k} \text {. }
$$

Ogni termine del determinante $\left(20_{5}\right)$ è dunque in valore assoluto minore di

$$
2 \frac{(\lambda n a)^{p(n-1)}}{2^{p(n-1)}(p(n-1)) !}\left(1+\frac{c}{n}\right)^{p(n-1)}
$$

e questa quantita tende a zero col crescere indefinito di $p$.

Il prodotto degli elementi della diagonale principalt converge assolutamente e tende ovviamente a zero.

Si deduce anche che $e$ assolutamente convergente la somma dei termini di ogni orizzontale.

Verifichiamo che è anche assolutamente convergente la somma dei termini di ciascuna verticale. Per $p=0$ si hanno i termini $a_{q \circ}(q=0,1,2, \ldots)$ della prima verticale e risulta

$$
\left|a_{q 0}\right|=2\left|J_{q}^{(0)}\right|=2\left|J_{q}(\lambda n a) \cdot J_{q}(\lambda c a)\right|<2 \frac{(\lambda n a)^{q}}{2^{q} q !} \frac{(\lambda c a)^{q}}{2^{q} q !},
$$

cioè, essendo $c<1$, si ha

$$
\left|a_{q_{0}}\right|<2 \frac{(\lambda n a)^{2 q}}{\left.2^{2 q}(q)\right)^{2}}, \quad(q=0,1,2 \ldots) .
$$

La somma dei termini della prima colonna è pertanto assolutamente convergente.

Per la colonna $p^{n a}$ generica considereremo separatamente i termini $a_{q p}=J_{q-p}^{(p(n-1))}(1, \lambda)+J_{-(p+q)}^{(p(n-1))}(1, \lambda)$, con $q \leq p$ (al disopra della diagonale principale), e quelli con $p \leq q \leq p n$, e con $q \geq p n$ che sono al disotto della diagonale principale.

In base ai risultati precedenti si deduce facilmente

$$
\begin{gathered}
\left|a_{q p}\right|<\frac{\left(\frac{\lambda n a}{2}\right)^{p(n+1)-2 q}}{(p n-q) !(p-q) !}+\frac{\left(\frac{\lambda n a}{-}\right)^{p(n+1)+2 q}}{(p n+q) !(p+q) !} \text {, per } q \leq p \\
\left|a_{q p}\right|<\frac{\left(\frac{\lambda n a}{2}\right)^{p(n-1)}}{(p n-q) !(q-p) !}+\frac{\left(\frac{\lambda n a}{2}\right)^{p(n+1)+2 q}}{(p n+q) !(p+q) !}, \text { per } p \leq q \leq p n \\
\left|a_{q p}\right|<\frac{\left(\frac{\lambda n a}{2}\right)^{2 q-p(n+1)}}{(q-p n) !(q-p) !}+\frac{\left(\frac{\lambda n a}{2}\right)^{p(n+1)+2 q}}{(p n+q) !(p+q) !} \text {, per } q \geq p n .
\end{gathered}
$$


Da queste relazioni segue orviamente che è assolutamente convergente la somma dei termini di ciascuna colonna del determinante $\left(20_{5}\right)$.

Segue inoltre che è assolutamente convergente la somma dei termini non diagonali, o, più largamente ancora, che è assolutamente convergente la serie $\sum_{0}^{\infty}{\stackrel{\infty}{\Sigma_{\gamma}}}_{\sigma^{\prime}}^{\infty} a_{q_{n}}$. Invero questa serie risulta maggiorata da una serie che ̀े ottenuta sommando termine a tormine due serie $S_{1}, S_{2}$ a termini positivi entrambe convergenti.

Nella serie $S_{1}$ indichiamo rispettivamente con $S_{1}{ }^{\prime}, S_{1}^{\prime \prime}, S_{1}{ }^{\prime \prime \prime}$ la somma dei termini per cui è $q \leq p, p \leq q \leq p n, q \geq p n$.

Per $q \leq p$, posto $k=p-q$, si ha

$$
\begin{aligned}
S_{1}^{\prime} & =\sum_{0}^{\infty} \sum_{0}^{p} \frac{\left(\frac{\lambda n a}{2}\right)^{p(n+1)-2 q}}{(p n-q) !(p-q) !}=\sum_{0}^{\infty} \sum_{0}^{p} \frac{\left(\frac{\lambda n a}{2}\right)^{p(n-1)+2 k}}{[p(n-1)+k] ! k !}< \\
& <\sum_{0}^{\infty} \sum_{0}^{p} \frac{\left(\frac{\lambda n a}{2}\right)^{p(n-1)+2 k}}{(p(n-1)) ! k !}<\sum_{0}^{\infty} \frac{\left(\frac{\lambda n a}{2}\right)^{p(n-1)}}{p !} \sum_{0}^{\infty} \frac{\left(\frac{\lambda n a}{2}\right)^{2 k}}{k !}< \\
& <e^{\left(\frac{\lambda n a}{2}\right)^{n-1}} \cdot e^{\left(\frac{\lambda n a}{2}\right)^{2}} .
\end{aligned}
$$

Per $p \leq q \leq p n$, posto $k=q-p$, risulta

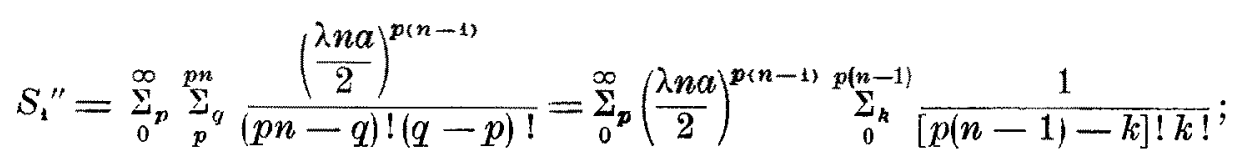

ma

$$
{\stackrel{p}{\Sigma_{k}}}_{n}^{p(n-1)} \frac{1}{[p(n-1)-k] ! k !}=\frac{1}{[p(n-1)] !}{\stackrel{p}{\sum_{k}}}^{p(n-1)} \frac{[p(n-1)] !}{[p(n-1)-k] ! k !}=\frac{2^{p(n-1)}}{[p(n-1)] !},
$$

percio

$$
S_{1}^{\prime \prime}=\sum_{0}^{\infty} \frac{(\lambda n a)^{p(n-1)}}{[p(n-1)] !}<\sum_{0}^{\infty} \frac{(\lambda n a)^{p(n-1,1}}{p !}=e^{(\lambda n a)^{n-1}}
$$

Per $q \geq p n$, ponendo $k=q-p n$, abbiamo

$$
\begin{aligned}
S_{1}^{\prime \prime \prime}= & \sum_{0}^{\infty} \sum_{p n}^{\infty} \frac{\left(\frac{\lambda n a}{2}\right)^{2 q-p(n+1)}}{(q-p n) !(q-p) !}=\sum_{0}^{\infty}, \sum_{0}^{\infty} \frac{\left(\frac{\lambda n a}{2}\right)^{p(n-1)+2 k}}{k ![p(n-1)+k] !}< \\
& <\sum_{0}^{\infty} \frac{\left(\frac{\lambda n a}{2}\right)^{p(n-1)}}{[p(n-1)] !} \sum_{0}^{\infty} \frac{\left(\frac{\lambda n a}{2}\right)^{2 k}}{k !}<e^{\left(\frac{\lambda n a}{2}\right)^{n-1}} \cdot e^{\left(\frac{\lambda n a}{z}\right)^{2}} .
\end{aligned}
$$


Per quanto riguarda la serie $S_{2}$, ponendo $p n+q=h, p+q=k$, essa risulta maggiorata dalla serie $\Sigma_{h k} \frac{\left(\frac{\lambda n a}{2}\right)^{h+k}}{h ! k !}$, che converge ed ha per somma $e^{\frac{\lambda n a}{q}} \cdot e^{\frac{\lambda n a}{q}}=e^{\lambda n a}$.

Il determinante $\left(20_{5}\right)$ è dunque convergente.

Con procedimento analogo si dimostra che sono convergenti gli altri determinanti di ordine infinito considerati in questo paragrafo.

9. Implicitamente abbiamo ammesso ancora che ciascuna delle equazioni determinanti nell'incognita $\lambda$ ammettesse infinite radici positive, che cioe vi siano infiniti autovalori del parametro $\lambda$. Ciò segue senz'altro dalla teoria delle equazioni integrali.

Invero il problema ai limiti di cui ci siamo occupati precedentemente pad essere ricondotto facilmente alla risoluzione di un'eqnazione integrale. Trasformiamo per questo $l^{\prime}$ equazione differenziale $\left(4_{4}\right)$ ponendo

$$
\xi=\rho \cos \varphi, \quad \eta=\rho \operatorname{sen} \varphi \text {. }
$$

Si ha allora

$$
F(u) \equiv \frac{\partial^{2} u}{\partial \xi^{2}}+\frac{\partial^{2} u}{\partial \eta^{2}}+\lambda^{2} n^{2} a^{2} R^{2}(\xi, \eta) \cdot u=0,
$$

ove $R^{2}(\xi, \eta)$ è cio che diventa l'espressione $\left(7_{i}\right)$ per effetto della sostituzione $\left(21_{5}\right)$. Il problema si traduce in quello di determinare soluzioni dell'equazione $\left(22_{5}\right)$ regolari entro il cerchio $\sigma$ con centro nell' origine $O$ degli assi $(\xi, \eta)$, e di raggio unitario, e che si annullano sul contorno circolare $s$. Insieme alla $\left(22_{5}\right)$ consideriamo la

$$
G(v) \equiv \frac{\partial^{2} v}{\partial \xi^{2}}+\frac{\partial^{2} v}{\partial \eta^{2}}=0
$$

e osserviamo che indicando con $\Delta_{2}=\frac{\partial}{\partial \xi^{2}}+\frac{\partial^{2}}{\partial \eta}$ l'operatore di LAPLACE nel piano, si ricava

$$
v F(u)-u G(v) \equiv v \Delta_{2} u-u \Delta_{2} v+\lambda^{2} n^{2} a^{2} R^{*}(\xi, \eta) u v=0 .
$$

La $v$ sia la funzione di GREEN relativa al cerchio, sia ciò armonica regolare in ogni punto $P(\xi, \eta)$ interno al cerchio $\sigma$, salvo nel punto $M\left(\xi^{\prime}, \eta^{\prime}\right)$ ove diventa infinita come $\log r$, essendo $r$ la distanza $P M$, e che si annulli sul contorno circolare $s$.

Se indichiamo con $\rho^{\prime}$ la distanza $O M$ e con $r_{1}$ la distanza del punto $P$ dal punto $M_{1}$, immagine di $M$ rispetto al cerchio $s$, risulta, com' ̀̀ noto,

$$
v=\log \frac{\rho^{\prime} r_{1}}{r}
$$


Circondando il punto $M$ con un cerchietto $s^{\prime}$ di centro $M$ che racchinde un' area $\sigma^{\prime}$, integriamo ambo i membri della $\left(24_{5}\right)$ estendendo l'integrazione al dominio $\sigma-\sigma^{\prime}$ limitato dalle circoferenze $s, s^{\prime}$; si ha cosl :

$$
\int_{\sigma-\sigma^{\prime}}\left(v \Delta_{2} u-u \Delta_{2} v\right) d \sigma+\lambda^{2} n^{2} \alpha^{2} \int_{\sigma \rightarrow \sigma^{\prime}} R^{2}(\xi, \eta) u v d \sigma=0 .
$$

Se $n$ è la normale esterna nei punti del contorno, si ha

$$
\int_{\sigma-\sigma^{\prime}}\left(v \Delta_{2} u-u \Delta_{2} v\right) d \sigma=\int_{s}\left(v \frac{d u}{d n}-u \frac{d v}{d n}\right) d s+\int_{s^{\prime}}\left(v \frac{d u}{d n}-u \frac{d v}{d n}\right) d s^{\prime}
$$

percio la formula precedente diventa

$$
\int_{s}\left(v \frac{d u}{d n}-u \frac{d v}{d n}\right) d s+\int\left(v \frac{d u}{d n}-u \frac{d v}{d n}\right) d s^{\prime}+\lambda^{2} n^{2} a^{2} \int_{\sigma-\sigma^{\prime}} R^{2}(\xi, \eta) u v d \sigma=0 .
$$

Ma la $u$ e la $v$ sono nulle sul contorno $s$, perciò passando al limite facendo tendere a zero il raggio del cerchio $s^{\prime}$ e osservando che risulta

$$
\lim \int_{s^{\prime}} v \frac{d u}{d n} d s^{\prime}=0, \quad \lim \int_{s^{\prime}} u \frac{d v}{d n} d s^{\prime}=2 \pi u(M)
$$

dalla $\left(25_{5}\right)$ si deduce

$$
-2 \pi u(M)+\lambda^{2} n^{2} a^{2} \int_{\sigma} R^{2}(\xi, \eta) \cdot \log \frac{\rho^{\prime} r_{1}}{r} \cdot u(\xi, \eta) d \xi d \eta=0 .
$$

Essendo $\rho, \varphi$ le coordinate polari di $P$ rispetto ad $O$, e $\rho^{\prime}, \varphi^{\prime}$ quelle di $M$, si ha

$$
\begin{gathered}
r=\sqrt{\rho^{2}+\rho^{\prime 2}-2 \rho \rho^{\prime} \cos \left(\varphi-\varphi^{\prime}\right)}, \\
r_{1}=\sqrt{\rho^{2}+\frac{1}{\rho^{\prime 2}}-2 \frac{\rho}{\rho^{\prime}} \cos \left(\varphi-\varphi^{\prime}\right)}=\frac{1}{\rho^{\prime}} \sqrt{1+\rho^{2} \rho^{\prime 2}-2 \rho \rho^{\prime}} \frac{\cos \left(\varphi-\varphi^{\prime}\right)}{}
\end{gathered}
$$

e dalla relazione precedente, passando a coordinate polari $\rho, \varphi$, segne:

$$
\begin{gathered}
u\left(\rho^{\prime}, \varphi^{\prime}\right)=\frac{1}{2 \pi} \lambda^{2} n^{2} a^{2} \int_{0}^{2 \pi} d \varphi \int_{0}^{1}\left[1+2 c \rho^{n-1} \cos (n-1) \varphi+\right. \\
\left.+c^{2} \rho^{(n-1)}\right] \log \sqrt{\frac{1+\rho^{2} \rho^{\prime 2}-2 \rho \rho^{\prime} \cos \left(\varphi-\varphi^{\prime}\right)}{\rho^{2}+\rho^{\prime 2}-2 \rho \rho^{\prime} \cos \left(\varphi-\varphi^{\prime}\right)}} \cdot u(\rho, \varphi) \cdot \rho d \rho .
\end{gathered}
$$

Dalla teoria delle equazioni integrali si sa che questa equazione ammette una infinità di valori caratteristici positivi del parameqro $\lambda^{2}$. Esiste dunque una infinità di numeri $\lambda_{i}$ (che si possono supporre positivi) a ciascuno dei $q$ uilli corrisponde una soluzione $u_{i}(\rho, \varphi)$ dell' equazione differenziale $\left(4_{1}\right)$ che si annulla per $\rho=1$. 


\section{§ 6. 2" Problema ai limiti. Autosoluzioni con derivata normale nulla al contorno epicicloidale.}

1. La ricerca di soluzioni $u(\rho, \wp)$ dell'equazione differenziale $\left(4_{1}\right)$, regolari entro il campo epicicloidale, la cui derivata normale si annulli al contorno, in virtù della $\left(17_{1}\right)$ equivale a cercare soluzioni regolari entro il cerchio di raggio $\rho=1$, sul quale è rappresentato conformemente il detto campo, e tali che $\frac{\partial u}{\partial \rho}=0$ per $\rho=1$.

Si utilizzerà allora nuovamente la soluzione generale $\left(12_{3}\right)$.

Da essa risulta che le equazioni determinanti degli autovalori del parametro $\lambda$ si otterranno da quelle del paragrafo precedente sostituendo alla

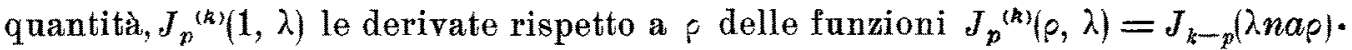
$\left.\cdot J_{p} \mid \lambda c a \rho^{\prime \prime}\right)$, calcolate per $\rho=1$, e che indicheremo oon $J_{p}{ }^{\prime(k)}(1, \lambda)$.

La soluzione generale soddisfacente a questa nuova condizione ai limiti assumerà ovviamente la stessa forma della $\left(19_{5}{ }^{\prime}\right)$, sarà somma cioè di autosoluzioni che indicheremo con

$$
E_{0}^{\prime(s)}, E_{0}^{(s(s)}, E_{k}^{(s)}, F_{k}{ }^{\prime(s)}, \quad(k=1,2, \ldots, n-2 ; s=1,2,3, \ldots)
$$

formanti un sistema fondamentale soddisfacenti a due a due a una relazione di ortogonalità analoga alla $\left(10_{5}\right)$.

2. Il problema ai limiti di cui è questione in questo paragrafo, permette di risolvere il problema della propagazione in un tubo epicicloidale indefinito di onde elettromagnetiche cosidette di tipo magnetico, per le quali cioè è nulla la componente $\mathcal{G}_{z}$ dol campo elettrico. Esso nel $\$ 1$, n. $3,1^{\circ}$ è stato ricondotto a determinare la componente $\mathfrak{H}_{z}$ del campo magnetico soddisfacente all'equazione differenziale $\left(4_{1}\right)$ con la condizione ai limiti $\frac{\partial \mathcal{H}_{z}}{\partial \rho}=0$, per $\rho=1$.

Esprimendo $\mathfrak{H}_{z}$ per mezzo delle funzioni epicicloidali ora definite avremo la soluzione generale

$$
\mathcal{H}_{z}=\underset{1}{\Sigma_{s}}\left\{M_{0}{ }^{(s)} E_{0}{ }^{(s)}+N_{0}{ }^{\prime(s)} E^{\prime *(s)}+\sum_{1}^{n-2}\left[M_{k}{ }^{(s)} E_{k}{ }^{(s)}+N_{k}^{\prime(s)} E_{k}{ }^{\prime *(s)}\right]\right\},
$$

ove le $M_{0}{ }^{(s)}, N_{0}^{\prime(s)}, M_{k}{ }^{\prime(s)}, N_{k}{ }^{\prime(s)}$ sono costanti arbitrarie.

3 La ricerca di soluzioni $u(\rho, \varphi)$ dell' equazione differenziale $\left(4_{1}\right)$, la cui derivata normal" si annulli al contorno epicicloidale, cioè per $\rho=1$, può essere ancora ricondotta alla risolnzione di un' equazione integrale.

Con le notazioni del paragrafo precedente n. 8, possiamo applicare nuovamente la formula $\left(25_{5}\right)$ ove la funzione $v$ è ora così definita: è armonica e 
regolare entro il cerchio $\sigma$, salvo nel punto $M$ ove diventa infinita come $\log r$, e la sua derivata normale si annulla sul contorno circolare $s$.

Ponendo $v=\log r+w$ con $w$ funzione armonica regolare entro il cerchio $\sigma$, avremo al contorno $s$,

$$
\frac{d w}{d n}=-\frac{d \log r}{d n}
$$

cioè, essendo $n$ la normale esterna, ricordando la $\left(26_{5}\right)$, sarà

$$
\frac{d w}{d n}=-\frac{\partial \log r}{\partial \rho}=-\left[\frac{\rho-\rho^{\prime} \cos \left(\varphi-\varphi^{\prime}\right)}{r^{2}}\right]_{\rho=1}
$$

orvero

$$
\frac{d w}{d n}=-\frac{1-\rho^{\prime} \cos \left(\varphi-\varphi^{\prime}\right)}{1+\rho^{\prime 2}-2 \rho^{\prime} \cos \left(\varphi-\varphi^{\prime}\right)}=-f\left(\varphi-\varphi^{\prime}\right)
$$

Si tratta allora di trovare una funzione armonica $w$ regolare entro il cerchio $\sigma$ la cui derivata normale esterna assuma sul contorno circolare $s$ il valore $-f\left(\varphi-\varphi^{\prime}\right)$, definito dalla relazione precedente. In virtù di una nota formula di DiNx $\left({ }^{5}\right)$ avremo

cioè

$$
w\left(\rho, \varphi ; \rho^{\prime}, \varphi^{\prime}\right)=\frac{1}{\pi} \int_{0}^{2 \pi} f(\tau) \log \sqrt{1+\rho^{2}-2 \rho \cos \left(\tau-\varphi+\varphi^{\prime}\right)} d \tau,
$$

$x^{\prime}\left(\rho, \varphi ; \rho^{\prime}, \varphi^{\prime}\right)=\frac{1}{\pi} \int_{0}^{2 \pi} \frac{1-\rho^{\prime} \cos \tau}{1+\rho^{\prime 2}-2 \rho^{\prime} \cos \tau} \log \sqrt{1+\rho^{2}-2 \rho \cos \left(\tau-\varphi+\varphi^{\prime}\right)} d \tau$.

Ritornando ora alla formula $\left(25_{5}\right)$, poichè al contorno circolare $s$ è $\frac{d u}{d n}=\frac{d v}{d n}=0$, passando al limite quando il raggio $r$ del cerchio $s^{\prime}$ tende a zero, osservando che si ha

$$
\lim \int_{s^{\prime}} v \frac{d u}{d n} d s^{\prime}=0, \quad \lim \int_{s^{\prime}} u \frac{d v}{d n} d s^{\prime}=-\lim \int_{s^{\prime}} u \frac{\partial v}{\partial r} d s^{\prime}=-2 \pi u(M),
$$

e ponendo

$$
G\left(\rho, \varphi ; \rho^{\prime}, \varphi^{\prime}\right)=-v=\log \frac{1}{r}-w\left(\rho, \varphi ; \rho^{\prime}, \varphi^{\prime}\right)
$$

si deduce

$u\left(\rho^{\prime}, \varphi^{\prime}\right)=\frac{1}{2 \pi} \lambda^{2} n^{2} a^{2} \int_{0}^{2 \pi} d \varphi \int_{0}^{1}\left[1+2 c \rho^{n-1} \cos (n-1) \varphi+c^{2} \rho^{2(n-t)}\right] G\left(\rho, \varphi ; \rho^{\prime}, \varphi^{\prime}\right) \cdot u(\rho, \varphi) \rho d \rho$ che è l'equazione integrale che volevamo stabilire.

(5) Tedi per es. M. Prcone, Appunti di Analisi superiore, $2^{a}$ edizione, vol. I, cap. II, $\S 2$, n. 15, Rondinella, editore, Napoli. 


\section{\& 7. 3o Problema ai limiti.}

Conduzione del calore in un cilindro epicicloidale indefinito.

1. Il problema della conduzione del calore in un corpo cilindrico omogeneo indefinito a sezione epicicloidale, che irradi calore nell'ambiente esterno a temperatura nulla, conduce, come si è visto nel $\S 1$, n. 4 , a considerare soluzioni dell'equazione differenziale $\left(4_{1}\right)$ soddisfacenti alla condizione

$$
\frac{1}{n a R} \frac{\partial u}{\partial \rho}+h u=0, \text { per } \rho=1,
$$

ove $R$ è definito dalla $\left(7_{4}\right)$ ed $h$ è una costante dipendente dal potere emissivo alla superficie del cilindro. Questa condizione si può scrivere anche

$$
n a h u+\frac{1}{\sqrt{1+c^{2}+2 c \cos (n-1) \varphi}} \frac{\partial u}{\partial \rho}=0, \text { per } \rho=1 .
$$

Dell' equazione differenziale $\left(4_{1}\right)$ incominciamo a considerare le soluzioni $E_{0}, E_{k},(k=1,2, \ldots, n-2)$, definite dalle relazioni $\left(21_{2}\right),\left(22_{2}\right)$.

Esse dovranno verificare la condizione $\left(1_{7}\right)$, arremo perciò

$\left(2_{\gamma}\right) n a h E_{k}+\frac{1}{\sqrt{1+c^{2}+2 c \cos (n-1) \varphi}} \frac{\partial E_{k}}{\partial \rho}=0$, per $\rho=1, \quad(k=0,1,2, \ldots, n-1)$. $\operatorname{cioè~}$

$$
\begin{aligned}
\left(3_{\gamma}\right) \quad & \operatorname{nah}\left[F_{0}(1, \lambda)+\sum_{1}^{\infty} F_{q(n-1)}(1, \lambda) \cos q(n-1) \varphi\right]+ \\
& +\frac{1}{\sqrt{1+c^{2}+2 c \cos (n-1) \varphi}}\left[F_{0}^{\prime}(1, \lambda)+\sum_{1}^{\infty} H_{q(n-1)}^{\prime}(1, \lambda) \cos q(n-1) \varphi\right]=0
\end{aligned}
$$

$\left(3_{7}^{\prime}\right) \operatorname{nah} \stackrel{\infty}{0}_{q}^{\infty} F_{q(n-1)+k}(1, \lambda) \cos (q(n-1)+k) \varphi+$

$$
\begin{array}{r}
+\frac{1}{\sqrt{1+c^{2}+2 c \cos (n-1) \varphi}} \sum_{0}^{\infty} F_{q(n-1)+k}^{\prime}(1, \lambda) \cos (q(n-1)+k) \varphi=0, \\
(k=1,2, \ldots, n-2) .
\end{array}
$$

nelle quali si sono contraddistinte con un apice le derivate delle funzioni $F$ rispetto a $\rho$, calcolate per $\rho=1$.

2. Dalla $\left(3_{7}\right)$, integrando ambo i membri rispetto a $\varphi$ da 0 a $2 \pi$, osservando che

$$
\int_{0}^{2 \pi} \frac{\cos (q(n-1)+k) \varphi}{\sqrt{1+c^{2}+2 c \cos (n-1) \varphi}} d \varphi=0, \quad \text { per } k=1,2, \ldots, n-2 ; q=0,1,2, \ldots
$$


e ponendo per brevità $\left({ }^{5}\right)$

$$
\Gamma_{q}=\frac{1}{2 \pi} \int_{0}^{2 \pi} \frac{\cos q(n-1) \varphi}{\sqrt{1+c^{2}+2 c \cos (n-1) \varphi}} d \varphi, \quad(q=0,1,2, \ldots)
$$

che sono costanti note, dipendenti dall'indice $q$ e da $c$, le quali restano invariate cambiando $q$ in $-q\left(\Gamma_{-q}=\Gamma_{q}\right)$, si ottiene l' equazione

$$
n a h F_{0}(1, \lambda)+\Gamma_{0} \cdot F_{0}^{\prime}(1, \lambda)+\sum_{1}^{\infty} \Gamma_{q} \cdot F_{q(n-1)}^{\prime}(1, \lambda)=0 .
$$

Moltiplichiamo ambo i membri della $\left(3_{7}\right)$ per $\cos l(n-1) \varphi,(l=0,1,2, \ldots)$, e integriamo rispetto a $\varphi$ da 0 a $2 \pi$. Poiche risulta

$$
\frac{1}{2 \pi} \int_{0}^{2 \pi} \frac{\cos q(n-1) \varphi \cdot \cos l(n-1) \varphi}{\sqrt{1+c^{2}+2 c \cos (n-1) \varphi}}=\frac{1}{2}\left(\Gamma_{q+l}+\Gamma_{q-i}\right)
$$

si ottengono le nuove equazioni

$$
\begin{aligned}
n a h F_{l(n-1)}(1, \lambda)+2 \Gamma_{l} F_{0}^{\prime}(1, \lambda)+\sum_{1}^{\infty}\left(\Gamma_{q+l}+\Gamma_{q-l}\right) F_{q(n-1)}^{\prime \prime}(1, \lambda) & =0, \\
& (l=1,2,3, \ldots) .
\end{aligned}
$$

Moltiplicando infine ambo i membri della $\left(3_{7}{ }^{\prime}\right)$ per $\cos (l(n+1)+k) \varphi$, $l=0,1,2, \ldots)$, e osservando che

$$
\begin{gathered}
\int_{0}^{2 \pi} \frac{\cos (q(n-1)+k) \varphi \cdot \cos (l(n-1)+k) \varphi}{\sqrt{1+c^{2}+2 c \cos (n-1) \varphi}} d \varphi= \\
\left.=\frac{1}{2} \int_{0}^{2 \pi}[\cos (q+l)(n-1)+2 k) \varphi+\cos (q-l)(n-1) \varphi\right] \frac{d \varphi}{\sqrt{1+c+2 c \cos (n-1) \varphi}}= \\
=\frac{1}{2} \int_{0}^{2 \pi} \frac{\cos (q-l)(n-1) \varphi}{\sqrt{1+c^{2}+2 c \cos (n-1) \varphi}} d \varphi=\pi \Gamma_{q-l},
\end{gathered}
$$

(5) Si osservi che in virtù della nota formula di Lipschitz

$$
\int_{0}^{\infty} J_{q}(s x) J_{q}(s y) d s=\frac{1}{2 \pi} \int_{0}^{2 \pi} \frac{\cos q \theta d \theta}{\sqrt{x^{2}+y^{2}-2 x y \cos \theta}},
$$

ponendo $(n-1) \varphi=\theta$, risulta anche:

$$
\Gamma_{q}=\frac{1}{2 \pi} \frac{1}{n-1} \int_{0}^{2 \pi i n-1)} \frac{\cos q \theta d: 1}{\sqrt{1+c^{2}+2 c \cos \theta}}=\frac{1}{2 \pi} \int_{0}^{2 \pi} \frac{\cos q^{\theta} d \theta}{\sqrt{1+c^{2}+2 c \cos \theta}}=(-1)^{q} \int_{0}^{\infty} J_{q}(s) J_{q}(s c) d s .
$$


sỉ ottengono le ulteriori equazioni

$$
\begin{aligned}
n a h F_{l(n-1)+k}(1, \lambda)+\stackrel{\Sigma}{q}_{q} \Gamma_{q-l} F_{q(n-1)+k}^{\prime}(1, \lambda)=0, & \\
& (l=0.1,2, \ldots ; k=1 \quad 2, \ldots, n-2) .
\end{aligned}
$$

3. Consideriamo ora dell'equazione differenziale $\left(4_{4}\right)$ le soluzioni $E_{0}{ }^{*}, E_{k}{ }^{*}$, $(k=1,2, \ldots, n-2)$, definite dalle $\left(7_{3}\right),\left(8_{3}\right)$. Imponendo che anche queste soluzioni verifichino la condizione $\left(1_{q}\right)$, che sia cioè :

$n a h E_{k}^{*}+\frac{1}{\sqrt{1+c^{2}+2 c \cos (n-1) \varphi}} \frac{\partial E_{k}^{*}}{\partial \rho}=0$, per $\rho=1, \quad(k=0,1,2, \ldots, n-2)$, con calcoli perfettamente analoghi a quelli del numero precedente si deducono le equazioni

$\left(\alpha^{*}\right) \quad n a h F_{l(n-1)}^{*}(1, \lambda)+\sum_{1}^{\infty}\left(\Gamma_{l-q}-\Gamma_{l+q}\right) F_{q(n-1)}^{* \prime}(1, \lambda)=0, \quad(\rho=1,2,3, \ldots)$,

$\left(\alpha_{1}^{*}\right) \quad n a h F_{l(n-1)+k}^{*}(1, \lambda)+\sum_{0}^{\infty} \Gamma_{q-l} F_{q(n-1)+k}^{*^{\prime}}(1, \lambda)=0$

$$
(l=0,1,2, \ldots ; k=1,2, \ldots, n-2),
$$

nelle quali al solito si sono contraddistinte con un apice le derivate rispetto a $\rho$ delle funzioni $F^{*}$ calcolate per $\rho=1$.

4. Sostituendo nelle equazioni $\left(\alpha_{0}\right),(\alpha),\left(\alpha_{1}\right),\left(\alpha^{*}\right),\left(\alpha_{1}^{*}\right)$ in luogo delle funzioni $F$ ed $F^{*}$, e delle loro derivate rispetto a $\rho$, i corrispondenti valori, e ponendo per brevità$$
f_{p(n-1)}^{(0)}(\lambda)=n a h J_{-p}^{(p(n-1))}(1, \lambda)+\Gamma_{0} J_{-p}^{(p(n-1))}(1, \lambda)+
$$
$+\sum_{1}^{\infty} \Gamma_{q}\left[J_{q-p}^{(p(n-1))}(1, \lambda)+J_{-(q+p)}^{(p(n-1))}(1, \lambda)\right]$

$f_{p(n-1)}^{(l)}(\lambda)=n a h\left[J_{l-p}^{(p(n-1)}(1, \lambda)+J_{-(l+p)}^{(p(n-1))}(1, \lambda)\right]+2 \Gamma_{l} J_{-p}^{\prime p(n-1))}(1, \lambda)+$ $+\sum_{1}^{\infty}\left(\Gamma_{q+l}+\Gamma_{q-i}\right)\left[J_{q-p}^{\prime(p(n-1))}(1, \lambda)+J_{-(q+p)}^{(p(n-1))}(1, \lambda)\right]$ $(l=1,2,3, \ldots)$,

$\left(5_{7}\right)$

$$
f_{p(n-1)+k}^{(l)}(\lambda)=n a h J_{l-p}^{(p(n-1)+k)}(1, \lambda)+\sum_{0}^{\infty} \Gamma_{q-l} J_{q-p}^{(p(n-1)+k)}(1, \lambda),
$$

$$
\begin{aligned}
g_{(p+1)(n-1-k}^{(l)}(\lambda)=n a h J_{-(p+l+1)}^{(p+1)(p-1)-k)}(1, \lambda)+ & { }_{0}^{\infty} \Gamma_{q}^{\infty} \Gamma_{q-l} J_{-(p+q+1)}^{(p+1)(n-1)-k)}(1, \lambda), \\
& (l=0,1,2, \ldots ; k=1,2, \ldots, n \quad 2),
\end{aligned}
$$

$\left(6_{\gamma}\right) \quad f_{p(n-1)}^{*(l)}(\lambda)=\operatorname{nah}\left[J_{l-p}^{(p(n-1))}(1, \lambda)-J_{-(n+p)}^{(p(n-1))}(1, \lambda)\right]+$

$$
\begin{array}{r}
+\sum_{1}^{\infty}\left(\Gamma_{l-q}-\Gamma_{l+q}\right)\left[J_{q-p}^{(p(n-1))}(1, \lambda)-J_{-(p+q)}^{(p(n-1))}(1, \lambda)\right] \\
(l=1,2,3, \ldots ; p=0,1,2, \ldots)
\end{array}
$$


le dette equazioni diventano

$$
{\stackrel{\infty}{\Sigma_{p}}}_{p}^{\infty} C_{p(n-1)} f_{p(n-1)}^{(0)}(\lambda)=0
$$

$$
\sum_{0}^{\infty} C_{p(n-1)} f_{p(n-1)}^{(n)}(\lambda)=0 \text {, }
$$

$$
(l=1,2,3, \ldots)
$$

$$
\begin{aligned}
& \stackrel{D}{v}_{p}^{\infty}\left[C_{p(n-1)+k} f_{p(n-1)+k}^{(l)}(\lambda)+C_{(p+1)(n-1)-k} g_{(p+1)(n-1)-k}^{(l)}(\lambda)\right]=0, \\
& (l=0,1,2, \ldots ; k=1,2, \ldots, n-2),
\end{aligned}
$$

$$
\sum_{0}^{\infty} C_{p(n-1)}^{*} f_{p(n-1)}^{*(l)}(\lambda)=0, \quad(l=1,2,3, \ldots)
$$

$$
\begin{aligned}
\sum_{0}^{\infty}\left[C_{p(n-1)+k}^{*} f_{p(n-1)+k}^{(l)}(\lambda)-C_{(p+1)(n-1)-k}^{*} g_{(p+1)(n-1)-k}^{(l)}(\lambda)\right]=0, \\
(l=0,1,2, \ldots ; k=1,2, \ldots, n-2) .
\end{aligned}
$$

Uguagliando a zero il determinante di ordine infinito costituito dai coefficienti delle costanti $C_{p_{(n-1)}}$ nelle $\left(\beta_{0}\right)$ e $(\beta)$, si ha l'equazione in $\lambda$

$$
\left\|f_{p(n-1)}^{(l)}(\lambda)\right\|=0, \quad\left(\begin{array}{lll}
p=0,1,2, \ldots & \text { nelle } & \text { orizzontali } \\
l=0,1,2, \ldots & \text { nelle verticali }
\end{array}\right),
$$

le cui radici positive, ordinate secondo valori crescenti, forniscono gli autovalori $\lambda_{\theta}{ }^{(\sigma)},(\sigma=1,2,3, \ldots$ del parametro $\lambda$.

Indicando con $D_{p(n-1)}^{(\sigma)}$ i complementi algebrici degli elementi dẹla prima riga del determinante $\left(7_{7}\right)$ calcolati per $\lambda=\lambda_{0}{ }^{(\sigma)}$, a meno di un fattore costante si può porre

$$
C_{p(n-1)}=D_{p(n-1)}^{(\sigma)}, \quad(p=0,1,2, \ldots ; \sigma=1,2,3, \ldots) .
$$

Le corrispondenti autofunzioni $F$ risultano

$$
\begin{aligned}
& F_{0}{ }^{(\sigma)}\left(\rho, \lambda_{0}(\sigma)\right)=\sum_{0}^{\infty} D_{p(n-1)}^{(\sigma)} J_{-p}^{(p(n-1))}\left(\rho, \lambda_{0}{ }^{(\sigma)}\right) \\
& F_{l(n-1)}^{(\sigma)}\left(\rho, \lambda_{0}^{(\sigma)}\right)=\sum_{0}^{\infty} D_{p(n-1)}^{(\sigma)}\left[J_{l-p}^{(p(n-1))}\left(\rho, \lambda_{0}^{(\sigma)}\right)+J_{-(l+p)}^{(p(n) 1)}\left(\lambda, \rho_{0}^{(\sigma)}\right)\right],(l=1,2,3, \ldots) .
\end{aligned}
$$

Analogamente dalle $\left(\beta_{1}\right)$ per ogni $k$ da 1 ad $n-2$, si deduce l'equazione in $\lambda$

$$
\left\|f_{p(n-1)+k}^{(l)}(\lambda), g_{(p+1)(n-1)-k}^{(l)}(\lambda)\right\|=0,\left(\begin{array}{l}
p=0,1,2, \ldots \text { nelle orizzontali } \\
l=0,1,2, \ldots \text { nelle verticali }
\end{array}\right) .
$$

Indicando con $\lambda_{k}^{(\sigma)},(\sigma=1,2,3, \ldots)$, le radici positive di essa, ordinate seconilo valori crescenti, e con $D_{p(n-1)+k}^{(\sigma)}, D_{(p+1)(n-1)-3}^{(\sigma)}$ i complementi algebrici degli elementi della prima riga del determinante $\left(9_{\eta}\right)$, calcolati per $\lambda=\lambda_{k}{ }^{(\sigma)}$, si può porre

$$
\left.O_{p(n-1)+k}=D_{p(n}^{(\sigma)} 1\right)+k, \quad C_{(p+1)(n-1)-k}=D_{(p+1)(n-1)-k}^{(\sigma)},
$$


e si hanno quindi le corrispondenti autofunzioni $F$ :

$$
\begin{gathered}
F_{l(n-1)+k}^{(\sigma)}\left(\rho, \lambda_{k}(\sigma)\right)=\sum_{0}^{\infty}\left[D_{p(n-1)+k}^{(\sigma)} J_{-p}^{(p(n-1)+k)}\left(\rho, \lambda_{k}^{(\sigma)}\right)+\right. \\
\left.+D_{(p+1)(n-1)-k}^{(\sigma)} J_{-(p+l+1)}^{(p+1)(n-1)-k)}\left(\rho, \lambda_{k}^{(\sigma)}\right)\right], \quad(l=0,1,2, \ldots ; k=1,2, \ldots, n-2) .
\end{gathered}
$$

Dell' equazione differenziale $\left(4_{4}\right)$ abbiamo pertanto le autosoluzioni

$$
E_{0}^{(\sigma)}\left(\rho, \varphi, \lambda_{0}{ }^{(\sigma)}\right)=F_{0}^{(\sigma)}\left(\rho, \lambda_{0}^{(\sigma)}\right)+\sum_{1}^{\infty} F_{l(n-1)}^{(\sigma)}\left(\rho, \lambda_{0}^{(\sigma)}\right) \cos q(n-1) \varphi
$$

$E_{k}(\sigma)\left(\rho, \varphi, \lambda_{k}{ }_{k}^{(\sigma)}\right)=\stackrel{\infty}{\Sigma}_{0}^{\infty} F_{l(n-1)+k}^{(\sigma)}\left(\rho . \lambda_{k}^{(\sigma)}\right) \cos (l(n-1)+k) \varphi,(k=1,2, \ldots, n-2)$, soddisfacenti alla condizione ai limiti $\left(1_{\eta}\right)$.

亡 da osservare che nel caso di $n$ dispari, per $k=\frac{n-1}{2}$, le equazioni $\left(\beta_{1}\right)$ diventano

$$
\stackrel{\infty}{\Sigma}_{0}^{\infty} C_{\left(p+\frac{1}{2}\right)(n-1)}\left[f_{\left(p+\frac{1}{2}\right)(n-1)}^{(l)}(\lambda)+g_{\left(p+\frac{1}{2}\right)(n-1)}^{(l,}(\lambda)\right]=0, \quad(l=0, \cdot 1,2, \ldots)
$$

con

$$
f_{\left(p+\frac{1}{2}\right)(n-1)}^{(l)}(\lambda)=n a h J_{l-p}^{\left(\left(p+\frac{l}{2}\right)(n-1)\right)}(1, \lambda)+\sum_{0}^{\infty} \Gamma_{q-l} J_{q-p}^{\prime\left(\left(p+\frac{1}{2}\right)(n-1)\right)}(1, \lambda)
$$

$$
g_{\left(p+\frac{1}{2}\right)(n-1)}^{(l)}(\lambda)=n a h J_{-(p+7+1)}^{\left(\left(p+\frac{1}{2}\right)(n-1)\right)}(1, \lambda)+\sum_{0}^{\infty} \Gamma_{q-l} J_{-(p+q+1)}^{\prime\left(\left(p+\frac{1}{2}\right)(n-1)\right)}(1, \lambda) .
$$

Corrispondentemente l'equazione in $\lambda$ risulta

$$
\left\|f_{\left(p+\frac{1}{2}\right)(n-1)}^{(l)}(\lambda)+g_{\left(p+\frac{1}{2}\right)(n-1)}^{(l)}(\lambda)\right\|=0, \quad(p=0,1,2, \ldots ; l=0,1,2, \ldots)
$$

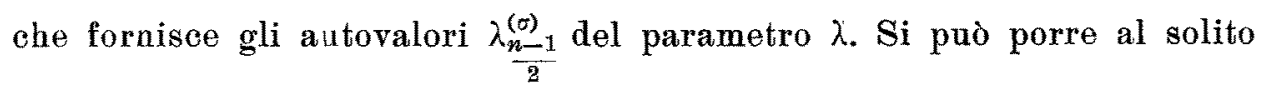

$$
C_{\left(p+\frac{1}{2}\right)(n-1)}=D_{\left(p+\frac{1}{2}\right)(n-1)}^{(\alpha)}
$$

ove $i$ secondi membri sono $i$ complementi algebrici degli elementi della prima riga del determinante $\left(9_{7}^{\prime}\right)$ calcolati per $\lambda=\frac{\lambda_{\frac{n-1}{2}}^{(\sigma)}}{2}$.

Si hanno quindi le autofunzioni corrispondenti

$$
\begin{aligned}
& F_{\left(l+\frac{1}{2}\right)(n-1,}^{(\sigma)}\left(\rho, \lambda_{\left.\frac{n-1}{2}\right)}^{(\sigma)}\right)=\sum_{0}^{\infty} D_{\left(p+\frac{1}{2}\right)(n-1)}^{(\sigma)}\left[J_{l-p}^{\left(\left(p+\frac{1}{2}\right)(n-1)\right)}\left(\rho, \lambda_{\frac{n-1}{2}}^{(\sigma)}\right)^{+}\right. \\
& \left.\left.+J_{-(p+i+1)}^{\left(\left(p+\frac{1}{2}\right)(n-1)\right)}\left(\rho, \lambda_{\frac{n-1}{2}}^{(\sigma)}\right)\right], \quad \quad l=0,1,2, . .,\right) .
\end{aligned}
$$


e le autosoluzioni

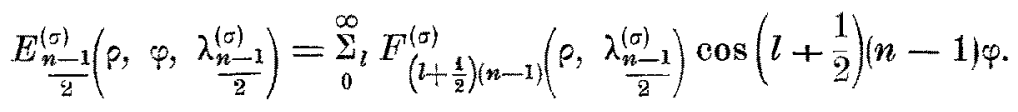

5. Dalle equazioni $\left(\beta^{*}\right)$ segue l'altra equazione determinante

$$
\left\|f_{p(n-1)}^{*(l)}(\lambda)\right\|=0, \quad(p=0,1,2, \ldots ; l=1,2,3, \ldots)
$$

le cui radici positive forniscono i nuovi autovalori $\lambda_{0} *(\sigma),(\sigma=1,2,3, \ldots)$.

Inoltre, essendo $D_{p(n-1)}^{*(\sigma)}$ i complementi algebrici dogli elementi della prima riga del determinante precedente, calcolati per $\lambda=\lambda_{\theta}{ }^{*(\sigma)}$ si può porre al solito

e si hanno le autofunzioni

$$
C_{p(n-1)}^{*}=D_{p(n-1)}^{*(\sigma)}
$$

$$
\begin{aligned}
& F_{l(n-1)}^{*(\sigma)}\left(\rho, \lambda_{0}{ }_{0}^{(\sigma)}\right)=\sum_{0}^{\infty}{ }_{p} D_{p(n-1)}^{*(\sigma)}\left[J_{l-p}^{(p(n-1))}\left(\rho, \lambda_{0} *(\sigma)\right)-J_{-(p+l)}^{(p(n-1))}\left(\rho, \lambda_{0} *(\sigma)\right)\right] \\
& (l=1,2,3, \ldots ; \sigma=0,1,2,3, \ldots) \text {. }
\end{aligned}
$$

Infine le equazioni $\beta_{1}^{*}$, per ogni $k$ da 1 ad $n-2$, danno luogo alla stessa equazione determinante $\left(9_{7}\right)$ e agli stessi autovalori $\lambda_{k}(\sigma)$ del parametro $\lambda$ considerati nel numero precedente.

Si può porre in questo caso

$$
C_{p(n-1)+k}^{*}=D_{p(n-1)+k}^{(\sigma)}, \quad-C_{(p+1)(n-1)-k}^{*}=D_{(p+1)(n-1)-k}^{(\sigma)},
$$

e si ha che le autofunzioni $F_{l(n-1)+k}^{*(\sigma)}$ coincidono con le autofunzioni $F_{l(n-1)+l k}^{(\sigma)}$ definite dalla $\left(10_{\eta}\right)$.

Del problema ai limiti di cui ci occupiamo in questo paragrafo abbiamo dunque le nuove autosoluzioni

$$
\begin{aligned}
& E_{0}^{*(\sigma)}\left(\rho, \varphi, \lambda_{0} *(\sigma)\right)=\sum_{1}^{\infty} F_{l(n-1)}^{*(\sigma)}\left(\rho, \lambda_{0} *(\sigma)\right) \operatorname{sen} l(n-1) \varphi \\
& \left.E_{k} *(\sigma)\left(\rho, \varphi, \lambda_{k c} *(\sigma)\right)=\sum_{0}^{\infty} F_{l(n-1)+k}^{(\sigma)}\left(\rho, \lambda_{k}^{(\sigma)}\right) \operatorname{sen} l(n-1)+k\right) \rho,
\end{aligned}
$$

Anche qui va osservato che nel caso di $n$ dispari, per $k=\frac{n-1}{2}$, le equazioni $\left(\beta_{1}^{*}\right)$ diventano

$$
\stackrel{\infty}{\leftrightarrows}_{0} C_{\left(p+\frac{1}{2}\right)(n-1)}^{*}\left[f_{\left(p+\frac{1}{2}\right)(n-1)}^{(l)}(\lambda)-g_{\left(p+\frac{1}{2}\right)(n-1)}^{(l)}(\lambda)\right]=0, \quad(l=0,1,2, \ldots)
$$

ove le funzioni $f_{\left(p+\frac{1}{2}\right)(n-1)}^{(l)}(\lambda), g_{\left(p+\frac{1}{2}\right)(n-1)}^{(l)}(\lambda)$ sono ancora definite dalle $\left(12_{7}\right)$. L' equazione determinante è in questo caso

$$
\left\|f_{\left(p+\frac{1}{2}\right)(n-1)}^{(l)}(\lambda)-g_{\left(p+\frac{1}{2}\right)(n-1)}^{(l)}(\lambda)\right\|=0, \quad(p=0,1,2, \ldots ; l=0,1,2, \ldots)
$$


le cui radici positive indichiamo con $\frac{\lambda_{\frac{2}{2}}^{*(\sigma)}}{2}(\sigma=1,2,3, \ldots)$. Ponendo come sempre

$$
C_{\left(p+\frac{1}{2}\right)(n-1)}^{*}=D_{\left(p+\frac{1}{2}\right)(n-1)}^{*(\sigma)} . \quad(p=0,1,2, \ldots)
$$

ove $i$ secondi membri sono i complementi algebrici degli elementi della prima riga del determinante $\left(14_{\gamma}\right)$, si hanno le autofunzioni corrispondenti

$$
F_{\left(n+\frac{1}{2}\right)(n-1)}^{*(\sigma)}\left(\rho, \lambda_{\frac{n-1}{2}}^{*(\sigma)}\right)=\sum_{0}^{\infty} D_{\left(p+\frac{1}{2}\right)(n-1)}^{*(\sigma)}\left[J_{l-p}^{\left(\left(p+\frac{1}{2}\right)(n-1)\right)}\left(\rho, \lambda_{\frac{n-1}{2}}^{*(\sigma)}\right)+J_{-(p+1+1)}^{\left(\left(p+\frac{1}{2}\right)(n-1)\right)}\left(\rho, \lambda \underset{\left.\frac{n-1}{2}\right)}{*(\sigma)}\right)\right]
$$

e quindi le autosoluzioni

$$
E_{\frac{n-1}{2}}^{*(\sigma)}\left(\rho, \varphi, \frac{\lambda_{n-1}^{*}}{2}\right)=\sum_{0}^{\infty} F_{\left(l+\frac{1}{2}\right)(n-1)}^{*(\sigma)}\left(\rho, \frac{\lambda_{n-1}^{*(\sigma)}}{2}\right) \operatorname{sen}\left(l+\frac{1}{2}\right)(n-1) \varphi
$$

6. Riunendo i risultati di questo paragrafo, abbiamo, del problema ai limiti considerato, la soluzione generale

$$
u(\rho, \varphi)=\Sigma_{1}^{\Sigma_{\sigma}}\left[P_{0}(\sigma) E_{0}(\sigma)+Q_{0}(\sigma) E_{0} *(\sigma)+\sum_{1}^{n-2}\left(P_{k}^{(\sigma)} E_{k}(\sigma)+Q_{k}(\sigma) E_{k} *(\sigma)\right]\right]
$$

cioè, nel caso di $n$ pari risulta

$$
\begin{gathered}
\left(16_{\eta}\right) u(\rho, \varphi)=\Sigma_{1} \Sigma_{\sigma}\left\{P_{0}(\sigma)\left[F_{0}^{(\sigma)}\left(\rho, \lambda_{0}(\sigma)\right)+\sum_{1}^{\infty} F_{l \mid n-1)}^{(\sigma)}\left(\rho, \lambda_{0}^{(\sigma)}\right) \cos l(n-1) \varphi\right]+\right. \\
+Q_{0}^{(\sigma)} \sum_{1}^{\infty} F_{l(n-1)}^{*(\sigma)}\left(\rho, \lambda_{0} *(\sigma)\right) \operatorname{sen} l(n-1) \varphi+ \\
\left.+\sum_{1}^{n-2} \sum_{0}^{\infty} F_{l(n-1)+k}^{(\sigma)}\left(\rho, \lambda_{k}(\sigma)\right)\left[P_{k}^{(\sigma)} \cos (l(n-1)+k) \varphi+Q_{k}(\sigma) \operatorname{sen}(l(n-1)+k) \varphi\right]\right\},
\end{gathered}
$$

ove le $P_{0}^{(\sigma)}, Q_{0}^{(\sigma)}, P_{k}^{(\sigma)}, Q_{k}^{(\sigma)}$ sono costanti arbitrarie.

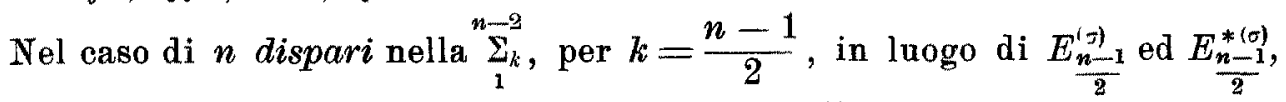
vanno rispettivamente poste le espressioni $\left(13_{7}\right)$ e $\left(15_{7}\right)$.

Con calcoli analoghi a quelli effettuati nel $\$ 5$, n. 3, si verifica che le autosoluzioni $E_{0}^{(\sigma)}, E_{k}^{(\sigma)}, E_{0} *[\sigma), E_{k} *(\sigma),(k=1,2, \ldots, n-2 ; \sigma=1,2,3, \ldots)$ verificano ancora a due a due la relazione di ortogonalità.

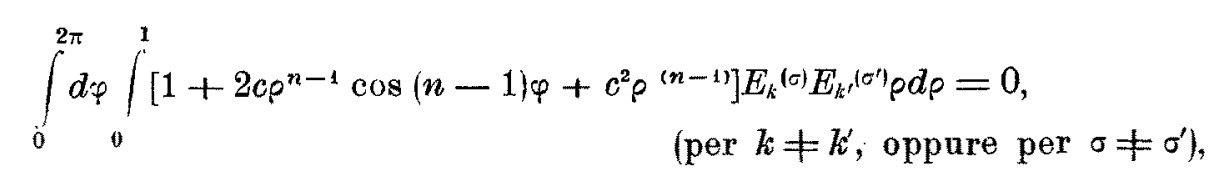

nelle quali le funzioni $E$ indicano indifferentemente le $E_{k}^{(\sigma)}$ e le $E_{l k} *|\sigma|$, con $k=0,1.2, \ldots, n \quad 2$. 
7. La soluzione generale $\left(16_{\tau}\right)$ dà il modo di risolvere il problema della conduzione del calore in un cilindro epicicloidale omogeneo indefinito che irradia calore nell'ambiente esterno a temperatura nulla, nell' ipotesi che sia assegnata la temperatura iniziale $u(\rho, \varphi)$, e questa sia rappresentabile mediante una serie del tipo $\left(16_{\gamma}\right)$.

In base ai risultati del $\S 1$, n. 4 , detta $U(\rho, \varphi, t)$ la temperatura in un punto interno al cilindro in un istante gene ico $t$, temperatura che si è sapposta indipendente dalla coordinata $z$, per $n$ pari sarà rappresentata dalla serie

$$
\begin{aligned}
U(\rho, \varphi, t) & =\Sigma_{1}\left\{P_{0}^{(\sigma)} E_{0}(\sigma) \cdot e^{-\left(\lambda_{0}(\sigma)\right)^{2} \cdot k t}+Q_{0}^{(\sigma)} E_{0} *(\sigma) \cdot e^{-\left(\lambda_{0}{ }^{(\sigma)}\right)^{2} \cdot k_{0} t}+\right. \\
& \left.+{ }_{1}^{n-\Sigma_{k}^{2}}\left[P_{k}(\sigma) E_{k}(\sigma)+Q_{k}(\sigma) E_{k} *(\sigma)\right] e^{\left.-\left(\lambda_{k}{ }^{(\sigma)}\right)\right)^{2} \cdot k_{0} t}\right\}
\end{aligned}
$$

che per $t=0$ si riduce alla $\left(16_{\eta}\right)$.

Essendo nota la funzione $u(\rho, \varphi)$, le costanti incognite $P_{0}{ }^{(\sigma)}, Q_{n}{ }^{(\sigma)}, P_{k}{ }^{(\sigma)}$, $Q_{k}{ }^{(\sigma)}$. si determinano dalla $\left(16_{7}\right)$ con noti procedimenti utilizzando le relazioni di ortogonalità che legano le funzioni $E$.

Si trova così :

$$
\begin{aligned}
& P_{k}(\sigma) \cdot \int_{0}^{2 \pi} d \varphi \int_{0}^{1} R^{2}(\rho, \varphi)\left(\left.E_{k}(\sigma)\right|^{2} \rho d \rho=\int_{0}^{2 \pi} d \varphi \int_{0}^{1} R^{2}(\rho, \varphi) E_{k}^{(\sigma)} \cdot u(\rho, \varphi) \cdot \rho d \rho,\right. \\
& Q_{k}(\sigma) \cdot \int_{0}^{2 \pi} d \varphi \int_{0}^{1} R^{2}(\rho, \varphi)\left(E_{k}^{*}{ }^{*}(\sigma)\right)^{2} \rho d \rho=\int_{0}^{2 \pi} d \varphi \int_{0}^{1} R^{2}(\rho, \varphi) E_{k}^{*}(\sigma) \cdot u(\rho, \varphi) \cdot \rho d \rho,
\end{aligned}
$$

ove $R^{2}(\varphi, \varphi)$ ha l'espressione $\left(7_{1}\right)$.

Nel caso di $n$ dispari nel secondo membro della $\left(17_{7}\right)$, il termine che si ha per $k=\frac{n-1}{2}$, risulta

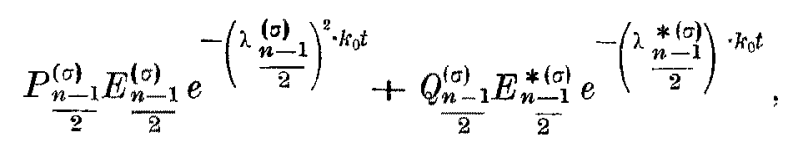

e le costanti $P_{\frac{n-1}{2}}^{(\sigma\}}, \frac{Q_{n-1}^{(\sigma)}}{\frac{2}{2}}$ saranno fornite da relazioni che si ottengono dalle $\left(18_{7}\right)$ ponendo $\frac{n-1}{2}$ al posto di $k$.

8. La ricerca di soluzioni dell' equazione differenziale $\left(4_{1}\right)$, con la condizione ai limiti $\left(1_{7}\right)$, si può ancora ricondurre alla risoluzione di un'equazione integrale. Con ciò risulterà dimostrata l'esistenza, da noi ammessa, di infiniti autovalori positivi del parametro $\lambda$. 
Essendo ora $n$ la normale esterna al cerchio di raggio $\rho=1$, su cui è rappresentato conformemente il campo epicicloidale, con le notazioni del $\$ 5$, n. 8 , la condizione $\left(1_{q}\right)$ si può scrivere

$$
\frac{d u}{d n}+n a h R \cdot u=0, \quad \text { (sopra } s \text { ). }
$$

In virtù di questa la formula $\left(25_{5}\right)$ diventa

$$
-\int_{s} u\left(\frac{d v}{d n}+n a h R v\right) d s+\int_{s^{\prime}}\left(v \frac{d u}{d n}-u \frac{d v}{d n}\right) d s^{\prime}+\lambda^{2} n^{2} a^{2} \int_{\sigma-\sigma^{\prime}} R u v d \sigma=0
$$

Se ora la $v$ è funzione armonica entro il cerchio $\sigma$, salvo nel punto $M$ ove diventa infinita come $\log r$, e sul contorno circolare $s$ verifica la condizione

$$
\frac{d v}{d n}+n a h R \cdot v=0
$$

la precedente, quando si passa al limite facendo tendere a zero il raggio della circonferenza $s^{\prime}$ di centro $M$, porge facilmente

$$
u(M)=\frac{1}{2 \pi} \lambda^{2} n^{2} a^{2} \int_{\sigma} R(P) v(P, M) u(P) d \sigma,
$$

che ò l'equazione integrale a cui abbiamo accennato.

Ponendo $v=g+w$, essendo con le notazioni del $\S 5, \mathrm{n} .8$,

$$
g=\log \frac{\rho^{\prime} r_{1}}{r},
$$

mentre $w$ è funzione armonica regolare entro il cerchio $\sigma$, poichè al contorno circolare $s$ è $g=0$, la condizione $\left(19_{7}\right)$ diventa

$$
\frac{d w}{d n}+n a h R \cdot w=-\frac{d g}{d n}, \quad \text { (sopra } s \text { ). }
$$

Quest' nltima questione $\mathrm{e}$ dunque ridotta a determinare la funzione $w$, armonica regolare entro il cerchio $\sigma$, che al contorno verifichi la condizione $\left(20_{7}\right)$, a per essa è noto il teorema di unicità e di esistenza. 